\title{
Generalized Halo Independent Comparison of Direct Dark Matter Detection Data
}

\author{
Eugenio Del Nobile ${ }^{1}{ }^{\dagger}$, Graciela Gelmini ${ }^{1}$ \% \\ PaOlo Gondolo ${ }^{2} *$ J J-Haeng HuH ${ }^{1}{ }^{17}$ \\ ${ }^{1}$ Department of Physics and Astronomy, UCLA, \\ 475 Portola Plaza, Los Angeles, CA 90095, USA \\ ${ }^{2}$ Department of Physics and Astronomy, University of Utah, \\ 115 S 1400 E Suite 201, Salt Lake City, UT 84112, USA
}

\begin{abstract}
We extend the halo-independent method to compare direct dark matter detection data, so far used only for spin-independent WIMP-nucleon interactions, to any type of interaction. As an example we apply the method to magnetic moment interactions.
\end{abstract}

delnobile@physics.ucla.edu

* gelmini@physics.ucla.edu

*paolo.gondolo@utah.edu

†jhhuh@physics.ucla.edu 


\section{Introduction}

Determining what the dark matter (DM), the most abundant form of matter in the Universe, consists of is one of the most fundamental open questions in physics and cosmology. Weakly interacting massive particles (WIMPs) are among the most experimentally sought after candidates. Four direct detection experiments, DAMA [1], CoGeNT [2,3], CRESST-II [4] and CDMSII-Si [5] have reported potential signals of WIMP DM, while all other direct detection searches have produced only upper bounds on interaction rates and annual modulation of the signal 6 -12].

An interesting way of comparing all these data, which circumvents the uncertainties in our knowledge of the local characteristics of the dark halo of our galaxy, is the 'halo-independent' comparison method [13 20]. The main idea of this method is that the interaction rate at one particular recoil energy $E_{\mathrm{R}}$ depends for any experiment on one and the same function $\eta\left(v_{\min }\right)$ of the minimum speed $v_{\text {min }}$ required for the incoming DM particle to cause a nuclear recoil with energy $E_{\mathrm{R}}$. The function $\eta\left(v_{\min }\right)$ depends only on the local characteristics of the dark halo of our galaxy. Thus, all rate measurements and bounds can be translated into measurements and bounds on the unique function $\eta\left(v_{\min }\right)$.

So far, this method was applied to the standard spin-independent (SI) WIMP-nucleus interaction only, although it could easily be applied to the standard spin-dependent (SD) interaction as well. For both SI and SD interactions, the differential scattering cross section has a $1 / v^{2}$ dependence on the speed $v$ of the DM particle. However, there are many other kinds of interactions with more general dependence on the DM particle velocity and on the nuclear recoil energy. Examples of these are: DM interacting through effective operators [21 32], WIMPs with electromagnetic couplings [33-36], in particular via a magnetic dipole moment 37 52], "Resonant DM" [53, 54], "Form factor DM" [55], "Anapole DM" [56]. While some of these interactions can be treated in a halo-independent fashion with trivial modifications of the method used so far for the SI interactions, this method cannot be applied to all of them, such as the magnetic dipole and anapole moment interactions, or resonant DM.

What forbids a trivial extension of the SI method in the case of magnetic dipole and anapole moment interactions is that the cross sections contain two different terms with different dependences on the DM particle speed $v$. When these terms are integrated over the velocity distribution to find the rate, 
instead of a unique function $\eta\left(v_{\text {min }}\right)$, each term has its own function of $v_{\text {min }}$ multiplied by its own detector dependent coefficient. It is thus impossible to translate a rate measurement or bound into only one of the two $v_{\min }$ functions contributing to the rate. Similarly, what forbids a trivial extension of the SI method to the case of "Resonant DM" is that the cross section for "Resonant DM" has a Breit-Wigner energy dependence with a shape that depends on the target nucleus. Thus each target has its own function of $v_{\min }$, and again it seems impossible to find one and the same common function analogous to

$\eta\left(v_{\min }\right)$ so that all rate measurements and bounds can be mapped onto it.

The aim of this paper is to extend the halo-independent analysis to all interactions circumventing the complications just mentioned. We find for any kind of interaction how to map all the rate measurements and bounds obtained with different experiments into a unique function of $v_{\min }$ that depends on the local characteristics of the dark halo of our galaxy only.

In Sec. 2 we fix our notation and recall the formalism for the haloindependent analysis as used so far for SI interactions. In Sec. 3 we present our generalized halo independent method, applicable to any type of interaction. We concentrate on elastic collisions, but present the formalism for inelastic collisions in Appendix A. Then in Sec. 4 we explain how we proceed to compare data in a halo independent manner, and in Secs. 5 and 6 we apply the method to Magnetic Dipole Moment DM (MDM). In Sec. 7 we present our concluding remarks.

\section{Halo-independent method - SI interactions}

The DM-nucleus differential scattering rate in counts $/ \mathrm{kg} /$ day $/ \mathrm{keV}$ for nuclear recoil energy $E_{\mathrm{R}}$ and target nuclide $T$, is

$$
\frac{\mathrm{d} R_{T}}{\mathrm{~d} E_{\mathrm{R}}}=\frac{\rho}{m} \frac{C_{T}}{m_{T}} \int_{v \geqslant v_{\min }\left(E_{\mathrm{R}}\right)} \mathrm{d}^{3} v f(\mathbf{v}, t) v \frac{\mathrm{d} \sigma_{T}}{\mathrm{~d} E_{\mathrm{R}}}\left(E_{\mathrm{R}}, \mathbf{v}\right) .
$$

Here $m$ is the DM particle mass, $m_{T}$ is the target nuclide mass, and $C_{T}$ is mass fraction of nuclide $T$ in the detector. The dependence of the rate on the local characteristics of the dark halo is contained in the local DM density $\rho$ and the DM velocity distribution in the Earth's frame $f(\mathbf{v}, t)$, which is modulated in time due to the Earth's rotation around the Sun. The distribution $f(\mathbf{v}, t)$ is normalized to $\int \mathrm{d}^{3} v f(\mathbf{v}, t)=1$. The minimum speed 
required for the incoming DM particle to cause a nuclear recoil with energy $E_{\mathrm{R}}$ is $v_{\min }$. For an elastic collision (see Appendix A for inelastic collisions),

$$
v_{\min }=\sqrt{\frac{m_{T} E_{\mathrm{R}}}{2 \mu_{T}^{2}}},
$$

where $\mu_{T}=m m_{T} /\left(m+m_{T}\right)$ is the WIMP-nucleus reduced mass.

To properly reproduce the recoil rate measured by experiments, we need to take into account the characteristics of the detector. Most experiments do not measure the recoil energy directly but rather a detected energy $E^{\prime}$, often quoted in keVee (keV electron-equivalent) or in photoelectrons. The uncertainties and fluctuations in the detected energy corresponding to a particular recoil energy are expressed in a (target nuclide and detector dependent) resolution function $G_{T}\left(E_{\mathrm{R}}, E^{\prime}\right)$ that gives the probability that a recoil energy $E_{\mathrm{R}}$ is measured as $E^{\prime}$. The resolution function is often but not always (the XENON experiments are a notable exception) approximated by a Gaussian distribution. It incorporates the mean value $\left\langle E^{\prime}\right\rangle=Q_{T} E_{\mathrm{R}}$, which depends on the energy dependent quenching factor $Q_{T}\left(E_{\mathrm{R}}\right)$, and the energy resolution $\sigma_{E_{\mathrm{R}}}\left(E^{\prime}\right)$. Moreover, experiments have a counting efficiency or cut acceptance $\epsilon\left(E^{\prime}\right)$, which also affects the measured rate. Thus the nuclear recoil rate in Eq. (1) must be convolved with the function $\epsilon\left(E^{\prime}\right) G_{T}\left(E_{\mathrm{R}}, E^{\prime}\right)$. The resulting differential rate as a function of the detected energy $E^{\prime}$ is

$$
\frac{\mathrm{d} R}{\mathrm{~d} E^{\prime}}=\epsilon\left(E^{\prime}\right) \sum_{T} \int_{0}^{\infty} \mathrm{d} E_{\mathrm{R}} G_{T}\left(E_{\mathrm{R}}, E^{\prime}\right) \frac{\mathrm{d} R_{T}}{\mathrm{~d} E_{\mathrm{R}}} .
$$

The rate within a detected energy interval $\left[E_{1}^{\prime}, E_{2}^{\prime}\right]$ follows as

$$
\begin{aligned}
& R_{\left[E_{1}^{\prime}, E_{2}^{\prime}\right]}(t)=\int_{E_{1}^{\prime}}^{E_{2}^{\prime}} \mathrm{d} E^{\prime} \frac{\mathrm{d} R}{\mathrm{~d} E^{\prime}} \\
&=\frac{\rho}{m} \sum_{T} \frac{C_{T}}{m_{T}} \int_{0}^{\infty} \mathrm{d} E_{\mathrm{R}} \int_{v \geqslant v_{\min }\left(E_{\mathrm{R}}\right)} \mathrm{d}^{3} v f(\mathbf{v}, t) v \frac{\mathrm{d} \sigma_{T}}{\mathrm{~d} E_{\mathrm{R}}}\left(E_{\mathrm{R}}, \mathbf{v}\right) \\
& \times \int_{E_{1}^{\prime}}^{E_{2}^{\prime}} \mathrm{d} E^{\prime} \epsilon\left(E^{\prime}\right) G_{T}\left(E_{\mathrm{R}}, E^{\prime}\right) .
\end{aligned}
$$

The differential cross section for the usual SI interaction is

$$
\frac{\mathrm{d} \sigma_{T}}{\mathrm{~d} E_{\mathrm{R}}}=\sigma_{T}^{\mathrm{SI}}\left(E_{\mathrm{R}}\right) \frac{m_{T}}{2 \mu_{T}^{2} v^{2}},
$$


with

$$
\sigma_{T}^{\mathrm{SI}}\left(E_{\mathrm{R}}\right)=\sigma_{p} \frac{\mu_{T}^{2}}{\mu_{p}^{2}}\left|Z_{T}+\left(A_{T}-Z_{T}\right) f_{n} / f_{p}\right|^{2} F_{\mathrm{SI}, T}^{2}\left(E_{\mathrm{R}}\right) .
$$

Here $Z_{T}$ and $A_{T}$ are respectively the atomic and mass number of the target nuclide $T, F_{\mathrm{SI}, T}\left(E_{\mathrm{R}}\right)$ is the nuclear spin-independent form factor, $f_{n}$ and $f_{p}$ are the effective DM couplings to neutron and proton, and $\mu_{p}$ is the DMproton reduced mass. Using this expression for the differential cross section, and changing integration variable from $E_{\mathrm{R}}$ to $v_{\text {min }}$ through Eq. (2), we can rewrite Eq. (4) as

$$
R_{\left[E_{1}^{\prime}, E_{2}^{\prime}\right]}^{\mathrm{SI}}(t)=\int_{0}^{\infty} \mathrm{d} v_{\min } \tilde{\eta}\left(v_{\min }, t\right) \mathcal{R}_{\left[E_{1}^{\prime}, E_{2}^{\prime}\right]}^{\mathrm{SI}}\left(v_{\min }\right),
$$

where the velocity integral $\tilde{\eta}$ is

$$
\tilde{\eta}\left(v_{\min }, t\right) \equiv \frac{\rho \sigma_{p}}{m} \int_{v \geqslant v_{\min }} \mathrm{d}^{3} v \frac{f(\mathbf{v}, t)}{v} \equiv \int_{v_{\min }}^{\infty} \mathrm{d}^{3} v \frac{\tilde{f}(\mathbf{v}, t)}{v},
$$

and we defined the response function $\mathcal{R}_{\left[E_{1}^{\prime}, E_{2}^{\prime}\right]}^{\mathrm{SI}}\left(v_{\min }\right)$ for WIMPS with SI interactions as

$$
\begin{aligned}
\mathcal{R}_{\left[E_{1}^{\prime}, E_{2}^{\prime}\right]}^{\mathrm{SI}}\left(v_{\min }\right) \equiv 2 v_{\min } \sum_{T} \frac{C_{T}}{m_{T}} \frac{\sigma_{T}^{\mathrm{SI}}\left(E_{\mathrm{R}}\left(v_{\min }\right)\right)}{\sigma_{p}} & \\
& \times \int_{E_{1}^{\prime}}^{E_{2}^{\prime}} \mathrm{d} E^{\prime} \epsilon\left(E^{\prime}\right) G_{T}\left(E_{\mathrm{R}}\left(v_{\min }\right), E^{\prime}\right) .
\end{aligned}
$$

Introducing the speed distribution

$$
\widetilde{F}(v, t) \equiv v^{2} \int \mathrm{d} \Omega_{v} \tilde{f}(\mathbf{v}, t)
$$

we can rewrite the $\tilde{\eta}$ function as

$$
\tilde{\eta}\left(v_{\min }, t\right)=\int_{v_{\min }}^{\infty} \mathrm{d} v \frac{\widetilde{F}(v, t)}{v} .
$$

Due to the revolution of the Earth around the Sun, the velocity integral $\tilde{\eta}\left(v_{\min }, t\right)$ has an annual modulation generally well approximated by the first terms of a harmonic series,

$$
\tilde{\eta}\left(v_{\min }, t\right) \simeq \tilde{\eta}^{0}\left(v_{\min }\right)+\tilde{\eta}^{1}\left(v_{\min }\right) \cos \left[\omega\left(t-t_{0}\right)\right],
$$


where $t_{0}$ is the time of the maximum of the signal and $\omega=2 \pi / \mathrm{yr}$. The unmodulated and modulated components $\tilde{\eta}^{0}$ and $\tilde{\eta}^{1}$ enter respectively in the definition of unmodulated and modulated parts of the rate,

$$
R_{\left[E_{1}^{\prime}, E_{2}^{\prime}\right]}(t)=R_{\left[E_{1}^{\prime}, E_{2}^{\prime}\right]}^{0}+R_{\left[E_{1}^{\prime}, E_{2}^{\prime}\right]}^{1} \cos \left[\omega\left(t-t_{0}\right)\right] .
$$

Once the WIMP mass and interactions are fixed, the functions $\tilde{\eta}^{0}\left(v_{\min }\right)$ and $\tilde{\eta}^{1}\left(v_{\text {min }}\right)$ are detector-independent quantities that must be common to all non-directional direct dark matter experiments. Thus we can map the rates measurements and bounds of different experiments into measurements of and bounds on $\tilde{\eta}^{0}\left(v_{\text {min }}\right)$ and $\tilde{\eta}^{1}\left(v_{\text {min }}\right)$ as functions of $v_{\text {min }}$.

Averages of the $\tilde{\eta}^{i}$ functions weighted by the response function $\mathcal{R}_{\left[E_{1}^{\prime}, E_{2}^{\prime}\right]}^{\mathrm{SI}}\left(v_{\min }\right)$ were compared in Refs. [15 and [17] with upper limits on $\tilde{\eta}^{i}$. The weighted averages practically coincide with the values assigned to the $\tilde{\eta}^{i}$ functions in Refs. [13], [14 and $[16]$ when, as assumed in those references, the energy interval is small enough that the differential rate, form factor and efficiency can be taken to be constant within the interval.

For experiments with putative DM signals, a rate $\hat{R}_{\left[E_{1}^{\prime}, E_{2}^{\prime}\right]}^{i}$ measured by an experiment in an energy interval $\left[E_{1}^{\prime}, E_{2}^{\prime}\right]$, translates into the average of $\tilde{\eta}^{i}\left(v_{\min }\right)$ in the corresponding $v_{\min }$ interval $\left[v_{\min , 1}, v_{\min , 2}\right]$ in which the response function $\mathcal{R}_{\left[E_{1}^{\prime}, E_{2}^{\prime}\right]}^{\mathrm{SI}}\left(v_{\text {min }}\right)$ is sufficiently different from zero,

$$
\overline{\tilde{\eta}_{\left[E_{1}^{\prime}, E_{2}^{\prime}\right]}^{i}} \equiv \frac{\hat{R}_{\left[E_{1}^{\prime}, E_{2}^{\prime}\right]}^{i}}{\int \mathrm{d} v_{\min } \mathcal{R}_{\left[E_{1}^{\prime}, E_{2}^{\prime}\right]}^{\mathrm{SI}}\left(v_{\min }\right)},
$$

with $i=0,1$ for the unmodulated and modulated component, respectively. The interval $\left[v_{\min , 1}, v_{\min , 2}\right]$ determines the width of the horizontal "error bar" in the $\left(v_{\min }, \tilde{\eta}\right)$ plane. In practice, following Ref. [14], for simplicity $v_{\min , 1}$ and $v_{\min , 2}$ were so far approximated by $v_{\min , 1}=v_{\min }\left(E_{1}^{\prime}-\sigma_{E_{\mathrm{R}}}\left(E_{1}^{\prime}\right)\right)$ and $v_{\min , 2}=$ $v_{\min }\left(E_{2}^{\prime}+\sigma_{E_{\mathrm{R}}}\left(E_{2}^{\prime}\right)\right)$. The vertical "error bar," unless otherwise indicated, showed the $68 \%$ confidence interval with Poissonian statistics.

To determine the upper bounds on the unmodulated part of $\tilde{\eta}$ set by experimental upper bounds on the unmodulated part of the rate, the procedure first outlined in Refs. 13, 14 was used. This limit exploits the fact that by definition $\tilde{\eta}^{0}$ is a non-increasing function of $v_{\min }$, thus the smallest possible $\tilde{\eta}^{0}\left(v_{\text {min }}\right)$ function passing by a fixed point $\left(v_{0}, \tilde{\eta}_{0}\right)$ in the $\left(v_{\min }, \tilde{\eta}\right)$ plane, is the downward step-function $\tilde{\eta}_{0} \theta\left(v_{0}-v_{\min }\right)$. In other words, among the functions passing by the point $\left(v_{0}, \tilde{\eta}_{0}\right)$, the downward step is the function yielding 
the minimum predicted number of events. Imposing this functional form in Eq. (7)

$$
R_{\left[E_{1}^{\prime}, E_{2}^{\prime}\right]}=\tilde{\eta}_{0} \int_{0}^{v_{0}} \mathrm{~d} v_{\min } \mathcal{R}_{\left[E_{1}^{\prime}, E_{2}^{\prime}\right]}^{\mathrm{SI}}\left(v_{\min }\right) .
$$

The upper bound $R_{\left[E_{1}^{\prime}, E_{2}^{\prime}\right]}^{\lim }$ on the unmodulated rate in an interval $\left[E_{1}^{\prime}, E_{2}^{\prime}\right]$ (usually at the $90 \%$ confidence level) is translated into an upper bound $\tilde{\eta}^{\lim }\left(v_{\min }\right)$ on $\tilde{\eta}^{0}$ at $v_{0}$ by

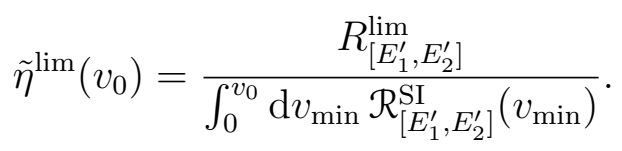

The upper bound so obtained is conservative in the sense that there are excluded functions $\tilde{\eta}^{0}\left(v_{\text {min }}\right)$ that nowhere exceed the limit. In other words, all functions $\tilde{\eta}^{0}\left(v_{\text {min }}\right)$ for which $\tilde{\eta}^{0}\left(v_{\text {min }}\right)>\tilde{\eta}^{\lim }\left(v_{\text {min }}\right)$ at some $v_{\text {min }}$ are excluded, but there are other excluded functions for which $\tilde{\eta}^{0}\left(v_{\min }\right) \leq \tilde{\eta}^{\lim }\left(v_{\min }\right)$ at all $v_{\min }[14]$.

The procedure just described does not assume any particular property of the dark halo. By making some assumptions, more stringent limits on the modulated part $\tilde{\eta}^{1}$ can be derived from the limits on the unmodulated part of the rate (see Refs. [18 20]), but we choose to proceed without making any assumption on the dark halo.

The procedure outlined in this section to compare data from different experiments in a halo independent way can only be applied when the differential cross section can be factorized into a velocity dependent term, independent of the detector (e.g. it must be independent of $m_{T}$ ), times a velocity independent term containing all the detector dependency. In the case of a more general form of the differential cross section, we can instead proceed as described in the following section.

\section{Generalized halo independent method}

Here we present a way of defining the response function $\mathcal{R}_{\left[E_{1}^{\prime}, E_{2}^{\prime}\right]}\left(v_{\text {min }}\right)$ in Eq. (7) that is valid for any type of interaction. Changing the order of the $\mathbf{v}$ 
and $E_{\mathrm{R}}$ integrations in Eq. (4), we have

$$
\begin{aligned}
R_{\left[E_{1}^{\prime}, E_{2}^{\prime}\right]}(t)=\frac{\rho \sigma_{\mathrm{ref}}}{m} \int_{0}^{\infty} \mathrm{d}^{3} v \frac{f(\mathbf{v}, t)}{v} \sum_{T} \frac{C_{T}}{m_{T}} \int_{0}^{E_{\mathrm{R}}^{\max }(v)} \mathrm{d} E_{\mathrm{R}} \frac{v^{2}}{\sigma_{\mathrm{ref}}} \frac{\mathrm{d} \sigma_{T}}{\mathrm{~d} E_{\mathrm{R}}}\left(E_{\mathrm{R}}, \mathbf{v}\right) \\
\times \int_{E_{1}^{\prime}}^{E_{2}^{\prime}} \mathrm{d} E^{\prime} \epsilon\left(E^{\prime}\right) G_{T}\left(E_{\mathrm{R}}, E^{\prime}\right) .
\end{aligned}
$$

Here $E_{\mathrm{R}}^{\max }(v) \equiv 2 \mu_{T}^{2} v^{2} / m_{T}$ is the maximum recoil energy a WIMP of speed $v$ can impart in an elastic collision to a target nucleus $T$ initially at rest. To make contact with the SI interaction method of the previous section, we have multiplied and divided by the factor $\sigma_{\text {ref }} / v^{2}$, where $\sigma_{\text {ref }}$ is a targetindependent reference cross section (i.e. a constant with the dimensions of a cross section) that coincides with $\sigma_{p}$ for SI interactions. In compact form, Eq. (17) reads

$$
R_{\left[E_{1}^{\prime}, E_{2}^{\prime}\right]}(t)=\int_{0}^{\infty} \mathrm{d}^{3} v \frac{\tilde{f}(\mathbf{v}, t)}{v} \mathcal{H}_{\left[E_{1}^{\prime}, E_{2}^{\prime}\right]}(\mathbf{v}),
$$

where in analogy with Eq. (8) we defined

$$
\tilde{f}(\mathbf{v}, t) \equiv \frac{\rho \sigma_{\text {ref }}}{m} f(\mathbf{v}, t),
$$

and we defined the "integrated response function" (the name stemming from Eq. (27))

$$
\begin{aligned}
\mathcal{H}_{\left[E_{1}^{\prime}, E_{2}^{\prime}\right]}(\mathbf{v}) \equiv \sum_{T} \frac{C_{T}}{m_{T}} \int_{0}^{E_{\mathrm{R}}^{\max }(v)} \mathrm{d} E_{\mathrm{R}} \frac{v^{2}}{\sigma_{\text {ref }}} & \frac{\mathrm{d} \sigma_{T}}{\mathrm{~d} E_{\mathrm{R}}}\left(E_{\mathrm{R}}, \mathbf{v}\right) \\
& \times \int_{E_{1}^{\prime}}^{E_{2}^{\prime}} \mathrm{d} E^{\prime} \epsilon\left(E^{\prime}\right) G_{T}\left(E_{\mathrm{R}}, E^{\prime}\right) .
\end{aligned}
$$

It will prove useful later to rewrite $\mathcal{H}_{\left[E_{1}^{\prime}, E_{2}^{\prime}\right]}$ by changing integration variable from $E_{\mathrm{R}}$ to $v_{\min }$ through Eq. (2), which yields

$$
\begin{aligned}
\mathcal{H}_{\left[E_{1}^{\prime}, E_{2}^{\prime}\right]}(\mathbf{v})=\sum_{T} \frac{C_{T}}{m_{T}} \frac{4 \mu_{T}^{2}}{m_{T}} \int_{0}^{v} \mathrm{~d} v_{\min } & v_{\min } \frac{v^{2}}{\sigma_{\text {ref }}} \frac{\mathrm{d} \sigma_{T}}{\mathrm{~d} E_{\mathrm{R}}}\left(E_{\mathrm{R}}\left(v_{\min }\right), \mathbf{v}\right) \\
& \times \int_{E_{1}^{\prime}}^{E_{2}^{\prime}} \mathrm{d} E^{\prime} \epsilon\left(E^{\prime}\right) G_{T}\left(E_{\mathrm{R}}\left(v_{\min }\right), E^{\prime}\right) .
\end{aligned}
$$


For simplicity, we only consider differential cross sections, and thus integrated response functions, that depend only on the speed $v=|\mathbf{v}|$, and not on the whole velocity vector. This is true if the DM flux and the target nuclei are unpolarized and the detection efficiency is isotropic throughout the detector, which is the most common case. With this restriction,

$$
R_{\left[E_{1}^{\prime}, E_{2}^{\prime}\right]}(t)=\int_{0}^{\infty} \mathrm{d} v \frac{\widetilde{F}(v, t)}{v} \mathcal{H}_{\left[E_{1}^{\prime}, E_{2}^{\prime}\right]}(v) .
$$

We now define the function $\tilde{\eta}(v, t)$ by

$$
\frac{\widetilde{F}(v, t)}{v}=-\frac{\partial \tilde{\eta}(v, t)}{\partial v},
$$

with $\tilde{\eta}(v, t)$ going to zero in the limit of $v$ going to infinity. This yields the usual definition of $\tilde{\eta}$ (see Eq. (11))

$$
\tilde{\eta}(v, t)=\int_{v}^{\infty} \mathrm{d} v^{\prime} \frac{\widetilde{F}\left(v^{\prime}, t\right)}{v^{\prime}} .
$$

Using Eq. (23) in Eq. (22) the energy integrated rate becomes

$$
R_{\left[E_{1}^{\prime}, E_{2}^{\prime}\right]}(t)=-\int_{0}^{\infty} \mathrm{d} v \frac{\partial \tilde{\eta}(v, t)}{\partial v} \mathcal{H}_{\left[E_{1}^{\prime}, E_{2}^{\prime}\right]}(v) .
$$

Integration by parts of Eq. (25) leads to an equation formally identical to Eq. (7) but which is now valid for any interaction,

$$
R_{\left[E_{1}^{\prime}, E_{2}^{\prime}\right]}(t)=\int_{0}^{\infty} \mathrm{d} v \tilde{\eta}(v, t) \mathcal{R}_{\left[E_{1}^{\prime}, E_{2}^{\prime}\right]}(v) .
$$

The response function is now defined as the derivative of the "integrated response function" $\mathcal{H}_{\left[E_{1}^{\prime}, E_{2}^{\prime}\right]}(v)$

$$
\mathcal{R}_{\left[E_{1}^{\prime}, E_{2}^{\prime}\right]}(v) \equiv \frac{\partial \mathcal{H}_{\left[E_{1}^{\prime}, E_{2}^{\prime}\right]}(v)}{\partial v} .
$$

Notice that the boundary term in the integration by parts of Eq. 25] is zero because the definition of $\mathcal{H}_{\left[E_{1}^{\prime}, E_{2}^{\prime}\right]}(\mathbf{v})$ in Eq. $\left[20\right.$ imposes that $\mathcal{H}_{\left[E_{1}^{\prime}, E_{2}^{\prime}\right]}(0)=0$ (since $E_{\mathrm{R}}^{\max }(0)=0$ ). 
In the rest of the paper we will only use the equations presented up to this point. However, it is interesting to notice that other expressions for the rate are possible. In fact, one can continue the integration by parts procedure of Eq. (25) to get a generalized version of Eq. (26). Defining iteratively

$$
\tilde{\eta}_{(k)}(v, t) \equiv k \int_{v}^{\infty} \mathrm{d} v^{\prime} \tilde{\eta}_{(k-1)}\left(v^{\prime}, t\right)
$$

for $k$ a positive integer, with $\tilde{\eta}_{(0)}(v, t) \equiv \tilde{\eta}(v, t)$, one can repeatedly integrate Eq. (26) by parts to get

$$
R_{\left[E_{1}^{\prime}, E_{2}^{\prime}\right]}(t)=\int_{0}^{\infty} \mathrm{d} v \tilde{\eta}_{(k)}(v, t) \mathcal{R}_{\left[E_{1}^{\prime}, E_{2}^{\prime}\right]}^{(k)}(v),
$$

where we also defined the response function of the $k$-th order

$$
\mathcal{R}_{\left[E_{1}^{\prime}, E_{2}^{\prime}\right]}^{(k)}(v) \equiv \frac{1}{k !} \frac{\partial^{k} \mathcal{R}_{\left[E_{1}^{\prime}, E_{2}^{\prime}\right]}(v)}{\partial v^{k}} .
$$

A derivation of this result is given in Appendix B. All boundary terms of the successive integrations by parts vanish because we have assumed that the response function and all of its derivatives vanish at $v=0$, a reasonable assumption since $v=0$ is below the threshold of any experiment. Going back to the 3-dimensional DM velocity distribution $\tilde{f}(\mathbf{v}, t)$, it is easy to prove (see Appendix B) that $\tilde{\eta}_{(k)}\left(v_{\text {min }}\right)$ is the so-called " $k$-th partial moment" of the function $\tilde{f}(\mathbf{v}) / v$, defined as

$$
\tilde{\eta}_{(k)}\left(v_{\min }, t\right)=\int_{v \geqslant v_{\min }}\left(v-v_{\min }\right)^{k} \frac{\tilde{f}(\mathbf{v}, t)}{v} \mathrm{~d}^{3} v .
$$

\section{Measurements of and bounds on $\tilde{\eta}$}

For the time being, similarly to what we did earlier for SI interactions, we want again to compare average values of the $\tilde{\eta}^{i}$ functions with upper limits. However, for a differential cross section with a general dependence on the DM velocity, it might not be possible to simply use Eq. (14) with $\mathcal{R}_{\left[E_{1}^{\prime}, E_{2}^{\prime}\right]}^{\mathrm{SI}}$ replaced by $\mathcal{R}_{\left[E_{1}^{\prime}, E_{2}^{\prime}\right]}$ to assign a weighted average of $\tilde{\eta}^{0}$ or $\tilde{\eta}^{1}$ to a finite $v_{\min }$ range. This may happen because the width of the response function $\mathcal{R}_{\left[E_{1}^{\prime}, E_{2}^{\prime}\right]}(v)$ in Eq. (27) at large $v_{\min }$ is dictated by the high speed behavior of the differential cross 
section, and it might even be infinite. For example, if $v^{2}\left(\mathrm{~d} \sigma_{T} / \mathrm{d} E_{\mathrm{R}}\right)$ goes as $v^{n}$, with $n$ a positive integer, for large $v$, then $\mathcal{H}_{\left[E_{1}^{\prime}, E_{2}^{\prime}\right]}(v)$ also goes as $v^{n}$ and $\mathcal{R}_{\left[E_{1}^{\prime}, E_{2}^{\prime}\right]}(v)$ goes as $v^{n-1}$ for large $v$. Thus, if $n \geqslant 1$, the response function $\mathcal{R}_{\left[E_{1}^{\prime}, E_{2}^{\prime}\right]}(v)$ does not vanish for large $v$. This implies that the denominator in Eq. (14) diverges.

However, we can regularize the behavior of the response function at large $v$ by using for example the function $v^{r} \tilde{\eta}(v)$ with integer $r \geqslant n$, instead of just $\tilde{\eta}(v)$. Since this new function is common to all experiments, we can use it to compare the data in $v_{\text {min }}$ space ${ }^{1}$ In fact, by multiplying and dividing the integrand in Eq. (26) by $v^{r}$, we can define the average of the function $v^{r} \tilde{\eta}(v)$ with weights $v^{-r} \mathcal{R}_{\left[E_{1}^{\prime}, E_{2}^{\prime}\right]}(v)$,

$$
\overline{v^{r} \tilde{\eta}_{\left[E_{1}^{\prime}, E_{2}^{\prime}\right]}^{i}}=\frac{\int_{0}^{\infty} \mathrm{d} v v^{r} \tilde{\eta}(v, t) v^{-r} \mathcal{R}_{\left[E_{1}^{\prime}, E_{2}^{\prime}\right]}(v)}{\int_{0}^{\infty} \mathrm{d} v v^{-r} \mathcal{R}_{\left[E_{1}^{\prime}, E_{2}^{\prime}\right]}(v)}
$$

With this definition,

$$
\overline{v^{r} \tilde{\eta}_{\left[E_{1}^{\prime}, E_{2}^{\prime}\right]}^{i}}=\frac{\hat{R}_{\left[E_{1}^{\prime}, E_{2}^{\prime}\right]}^{i}}{\int_{0}^{\infty} \mathrm{d} v v^{-r} \mathcal{R}_{\left[E_{1}^{\prime}, E_{2}^{\prime}\right]}(v)} .
$$

Notice that exploiting the definition of $\mathcal{R}_{\left[E_{1}^{\prime}, E_{2}^{\prime}\right]}$ in Eq. (27), we can write this relation in terms of $\mathcal{H}_{\left[E_{1}^{\prime}, E_{2}^{\prime}\right]}$ instead of $\mathcal{R}_{\left[E_{1}^{\prime}, E_{2}^{\prime}\right]}$ as

$$
\overline{v^{r} \tilde{\eta}_{\left[E_{1}^{\prime}, E_{2}^{\prime}\right]}^{i}}=\frac{\hat{R}_{\left[E_{1}^{\prime}, E_{2}^{\prime}\right]}^{i} \int_{0}^{\infty} \mathrm{d} v v^{-r-1} \mathcal{H}_{\left[E_{1}^{\prime}, E_{2}^{\prime}\right]}(v)}{},
$$

where in the integration by parts the finite term $\left[v^{-r} \mathcal{H}_{\left[E_{1}^{\prime}, E_{2}^{\prime}\right]}(v)\right]_{0}^{\infty}$ vanishes since by assumption $r$ has been appropriately chosen to regularize the integral of $v^{-r} \mathcal{R}_{\left[E_{1}^{\prime}, E_{2}^{\prime}\right]}(v)$, i.e. $v^{-r} \mathcal{H}_{\left[E_{1}^{\prime}, E_{2}^{\prime}\right]}(v) \rightarrow 0$ as $v \rightarrow \infty$.

Eqs. (33) or (34) allow to translate rate measurements in a detected energy interval $\left[E_{1}^{\prime}, E_{2}^{\prime}\right]$ into averaged values of $v^{r} \tilde{\eta}(v)$ in a finite $v_{\text {min }}$ interval $\left[v_{\min , 1}, v_{\min , 2}\right]$. This is now the interval outside which the integral of the new response function $v^{-r} \mathcal{R}_{\left[E_{1}^{\prime}, E_{2}^{\prime}\right]}(v)$ (and not of $\mathcal{R}_{\left[E_{1}^{\prime}, E_{2}^{\prime}\right]}(v)$ ) is negligible. We choose to use $90 \%$ central quantile intervals, i.e. we determine $v_{\min , 1}$ and

\footnotetext{
${ }^{1}$ While any other function that goes to zero fast enough would be equally good to regularize $\mathcal{R}_{\left[E_{1}^{\prime}, E_{2}^{\prime}\right]}(v)$, like for instance an exponentially decreasing function, we have chosen the power law $v^{-r}$ because it does not require the introduction of an arbitrary $v$ scale in the problem.
} 
$v_{\min , 2}$ such that the area under the function $v^{-r} \mathcal{R}_{\left[E_{1}^{\prime}, E_{2}^{\prime}\right]}(v)$ to the left of $v_{\min , 1}$ is $5 \%$ of the total area, and the area to the right of $v_{\min , 2}$ is also $5 \%$ of the total area. In practice, the larger the value of $r$, the smaller is the width of the $\left[v_{\min , 1}, v_{\min , 2}\right]$ interval, designated by the horizontal "error bar" of the crosses in the $\left(v_{\min }, \tilde{\eta}\right)$ plane. However, $r$ cannot be chosen arbitrarily large, because large values of $r$ give a large weight to the low velocity tail of the $\mathcal{R}_{\left[E_{1}^{\prime}, E_{2}^{\prime}\right]}(v)$ function, and this tail depends on the low energy tail of the resolution function $G_{T}\left(E_{\mathrm{R}}, E^{\prime}\right)$ in Eq. (20), which is never well known. Therefore too large values of $r$ make the procedure very sensitive to the way in which the tails of the $G_{T}\left(E_{\mathrm{R}}, E^{\prime}\right)$ function are modeled. This is explained in more detail in Sec. 6. (see also Fig. 1), where we use this procedure for a particular interaction. In the figures, the horizontal placement of the vertical bar in the crosses corresponds to the maximum of $v^{-r} \mathcal{R}_{\left[E_{1}^{\prime}, E_{2}^{\prime}\right]}(v)$. The extension of the vertical bar, unless otherwise indicated, shows the $1 \sigma$ interval around the central value of the measured rate.

The upper limit on the unmodulated part of $v^{r} \tilde{\eta}$ is simply $v^{r} \tilde{\eta}^{\lim }(v)$, where $\tilde{\eta}^{\lim }(v)$ is computed as described at the end of Sec. 2 by using a downward step-function $\tilde{\eta}_{0} \theta\left(v_{0}-v_{\text {min }}\right)$ for $\tilde{\eta}^{0}\left(v_{0}\right)$ to determine the maximum value of the step $\tilde{\eta}_{0}$. Given the definition of the response function $\mathcal{R}$ in the general case in terms of $\mathcal{H}$, Eq. (27), the downward step function choice for $\tilde{\eta}^{0}$ yields

$$
R_{\left[E_{1}^{\prime}, E_{2}^{\prime}\right]}=\tilde{\eta}_{0} \int_{0}^{v_{0}} \mathrm{~d} v_{\min } \mathcal{R}_{\left[E_{1}^{\prime}, E_{2}^{\prime}\right]}\left(v_{\min }\right)=\tilde{\eta}_{0} \mathcal{H}_{\left[E_{1}^{\prime}, E_{2}^{\prime}\right]}\left(v_{0}\right),
$$

From this equation we find the maximum value of $\tilde{\eta}_{0}$ at $v_{0}$ allowed by the experimental upper limit on the unmodulated rate $R_{\left[E_{1}^{\prime}, E_{2}^{\prime}\right]}^{\lim }$,

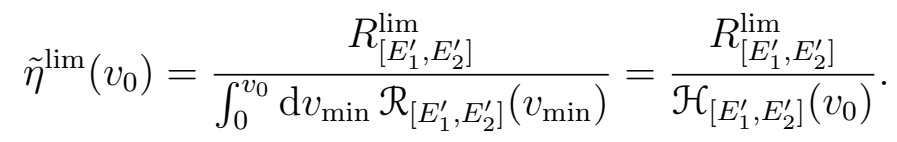

In the figures, rather than drawing the new averages $\overline{v^{r} \tilde{\eta}^{i}}$ and the limits $v^{r} \tilde{\eta}^{\lim }(v)$, we prefer to draw $v^{-r} \bar{v}^{r} \tilde{\eta}^{i}$ and $\tilde{\eta}^{\lim }(v)$, so that a comparison can be easily made with the previous literature on the SI halo-independent method.

\section{Magnetic-dipole dark matter (MDM)}

We now apply our new generalized method to a Dirac fermion DM candidate that interacts only through a magnetic dipole moment $\lambda_{\chi}$, the so-called 
magnetic-dipole dark matter (MDM) [37, 52],

$$
\mathscr{L}_{\text {int }}=\frac{\lambda_{\chi}}{2} \bar{\chi} \sigma_{\mu \nu} \chi F^{\mu \nu}
$$

The differential cross section for scattering of an MDM with a target nucleus is

$$
\begin{aligned}
\frac{\mathrm{d} \sigma_{T}}{\mathrm{~d} E_{\mathrm{R}}}=\alpha \lambda_{\chi}^{2}\left\{Z _ { T } ^ { 2 } \frac { m _ { T } } { 2 \mu _ { T } ^ { 2 } } \left[\frac{1}{v_{\min }^{2}}-\right.\right. & \left.\frac{1}{v^{2}}\left(1-\frac{\mu_{T}^{2}}{m^{2}}\right)\right] F_{\mathrm{SI}, T}^{2}\left(E_{\mathrm{R}}\left(v_{\min }\right)\right) \\
& \left.+\frac{\hat{\lambda}_{T}^{2}}{v^{2}} \frac{m_{T}}{m_{p}^{2}}\left(\frac{J_{T}+1}{3 J_{T}}\right) F_{\mathrm{M}, T}^{2}\left(E_{\mathrm{R}}\left(v_{\min }\right)\right)\right\} .
\end{aligned}
$$

Here $\alpha=e^{2} / 4 \pi$ is the electromagnetic fine structure constant, $m_{p}$ is the proton mass, $J_{T}$ is the spin of the target nucleus, and $\hat{\lambda}_{T}$ is the magnetic moment of the target nucleus in units of the nuclear magneton $e /\left(2 m_{p}\right)=0.16 \mathrm{GeV}^{-1}$. The first term corresponds to the dipole-nuclear charge coupling, and the corresponding charge form factor coincides with the usual spin-independent nuclear form factor $F_{\mathrm{SI}, T}\left(E_{\mathrm{R}}\right)$. We take it to be the Helm form factor [57] normalized to $F_{\mathrm{SI}, T}(0)=1$. The second term, which we call "magnetic", corresponds to the coupling of the DM magnetic dipole to the magnetic field of the nucleus, and the corresponding nuclear form factor is the nuclear magnetic form factor $F_{\mathrm{M}, T}\left(E_{\mathrm{R}}\right)$. This magnetic form factor is not identical to the spin form factor that accompanies SD interactions, in that the magnetic form factor includes the magnetic currents due to the orbital motion of the nucleons in addition to the intrinsic nucleon magnetic moments (spins).

For the light WIMPs we consider in the following, the magnetic term is negligible for all the target nuclei we consider except Na. This term is more important for lighter nuclei, such as $\mathrm{Na}$ and $\mathrm{Si}$, but $\mathrm{Si}$ has a very small magnetic dipole moment. The nuclear magnetic moment of ${ }^{23} \mathrm{Na}$ is $\hat{\lambda}_{\mathrm{Na}}=2.218$. We took the magnetic form factor $F_{\mathrm{M}, \mathrm{Na}}^{2}(q)$ from Fig. 31 of Ref. [58], which shows the "transverse form factor" $F_{\mathrm{T}}^{2}(q)$ for ${ }^{23} \mathrm{Na}$, defined as $F_{T}^{2}(q)=q^{2} F_{\mathrm{M}, T}^{2}(q)\left(\hat{\lambda}_{T}^{2} / 8 \pi m_{N}^{2}\right)\left(J_{T}+1\right) /\left(3 J_{T}\right)$. Here $q=\sqrt{2 m_{T} E_{\mathrm{R}}}$, and $m_{N}$ is the nucleon mass. We obtain $F_{\mathrm{M}, T}^{2}(q)$ by dividing $F_{\mathrm{T}}^{2}(q)$ by $q^{2}$ and normalizing it to $F_{\mathrm{M}, \mathrm{Na}}^{2}(0)=1$. The result is fitted by the approximate functional form $F_{\mathrm{M}, \mathrm{Na}}^{2}(q)=\left(1-1.15845 q^{2}+0.903442 q^{4}\right) \exp \left(-2.30722 q^{2}\right)$, where $q$ is in units of $\mathrm{fm}^{-1}$.

The spin-independent part of the differential cross section has two terms, one proportional to $1 / v_{\min }^{2}$ and another with a $1 / v^{2}$ dependence. The magnetic term also has a $1 / v^{2}$ dependence. Notice here the difficulty that our 
generalized method circumvents: had we proceeded with the same usual method to compute the rate used to get to Eq. (7), we would have obtained two terms in the rate each containing a different function of $v_{\text {min }}$ multiplied by detector dependent coefficients. It would have been impossible in this way to translate a rate measurement or bound into only one of the two $v_{\text {min }}$ functions.

Notice that the function $\mathcal{H}_{\left[E_{1}^{\prime}, E_{2}^{\prime}\right]}(v)$ has in this case a $v^{2}$ dependence for large values of $v$, with $\mathcal{R}_{\left[E_{1}^{\prime}, E_{2}^{\prime}\right]}(v)$ scaling as $v$. More precisely we have

$$
\begin{aligned}
& \mathcal{H}_{\left[E_{1}^{\prime}, E_{2}^{\prime}\right]}(v)=2 \sum_{T} \frac{C_{T}}{m_{T}} \int_{0}^{v} \mathrm{~d} v_{\text {min }} v_{\text {min }} \\
& \times\left\{Z_{T}^{2}\left[\frac{v^{2}}{v_{\text {min }}^{2}}-\left(1-\frac{\mu_{T}^{2}}{m^{2}}\right)\right] F_{\mathrm{SI}, T}^{2}\left(E_{\mathrm{R}}\left(v_{\min }\right)\right)+\hat{\lambda}_{T}^{2} \frac{2 \mu_{T}^{2}}{m_{p}^{2}}\left(\frac{J_{T}+1}{3 J_{T}}\right) F_{\mathrm{M}, T}^{2}\left(E_{\mathrm{R}}\left(v_{\min }\right)\right)\right\} \\
& \times \int_{E_{1}^{\prime}}^{E_{2}^{\prime}} \mathrm{d} E^{\prime} \epsilon\left(E^{\prime}\right) G_{T}\left(E_{\mathrm{R}}\left(v_{\min }\right), E^{\prime}\right),
\end{aligned}
$$

where we defined $\sigma_{\text {ref }} \equiv \alpha \lambda_{\chi}^{2}$. As a consequence,

$$
\begin{aligned}
& \mathcal{R}_{\left[E_{1}^{\prime}, E_{2}^{\prime}\right]}(v)=2 v \sum_{T} \frac{C_{T}}{m_{T}} \int_{0}^{\infty} \mathrm{d} v_{\min } \\
& \times\left[\left(Z_{T}^{2} \frac{\mu_{T}^{2}}{m^{2}} F_{\mathrm{SI}, T}^{2}\left(E_{\mathrm{R}}\left(v_{\min }\right)\right)+\hat{\lambda}_{T}^{2} \frac{2 \mu_{T}^{2}}{m_{p}^{2}}\left(\frac{J_{T}+1}{3 J_{T}}\right) F_{\mathrm{M}, T}^{2}\left(E_{\mathrm{R}}\left(v_{\min }\right)\right)\right) \delta\left(v-v_{\min }\right)\right. \\
& \left.\quad+\frac{2}{v_{\text {min }}} \theta\left(v-v_{\text {min }}\right) F_{\mathrm{SI}, T}^{2}\left(E_{\mathrm{R}}\left(v_{\text {min }}\right)\right)\right] \int_{E_{1}^{\prime}}^{E_{2}^{\prime}} \mathrm{d} E^{\prime} \epsilon\left(E^{\prime}\right) G_{T}\left(E_{\mathrm{R}}\left(v_{\min }\right), E^{\prime}\right) .
\end{aligned}
$$

The denominator of Eq. (33) is therefore

$$
\begin{gathered}
\int \mathrm{d} v v^{-r} \mathcal{R}_{\left[E_{1}^{\prime}, E_{2}^{\prime}\right]}(v)=2 \sum_{T} \frac{C_{T}}{m_{T}} \int_{0}^{\infty} \mathrm{d} v_{\min } v_{\min }^{-r+1} \\
\times\left[Z_{T}^{2}\left(\frac{\mu_{T}^{2}}{m^{2}}+\frac{2}{r-2}\right) F_{\mathrm{SI}, T}^{2}\left(E_{\mathrm{R}}\left(v_{\min }\right)\right)+\hat{\lambda}_{T}^{2} \frac{2 \mu_{T}^{2}}{m_{p}^{2}}\left(\frac{J_{T}+1}{3 J_{T}}\right) F_{\mathrm{M}, T}^{2}\left(E_{\mathrm{R}}\left(v_{\min }\right)\right)\right] \\
\int_{E_{1}^{\prime}}^{E_{2}^{\prime}} \mathrm{d} E^{\prime} \epsilon\left(E^{\prime}\right) G_{T}\left(E_{\mathrm{R}}\left(v_{\min }\right), E^{\prime}\right),
\end{gathered}
$$

where $r$ can be any number larger than 2 . 


\section{Data comparison for MDM}

The experimental data sets we consider are the following.

$D A M A$. We read the modulation amplitudes from Fig. 6 of Ref. [1]. We consider scattering off sodium only, since the iodine component is under threshold for low mass WIMPs and a reasonable local Galactic escape velocity. We show results for one single value of the $\mathrm{Na}$ quenching factor: $Q_{\mathrm{Na}}=0.30$. No channeling is included, as per Refs. [59,60].

CoGeNT. We use the list of events, quenching factor, efficiency, exposure times and cosmogenic background given in the 2011 CoGeNT data release [61]. We separate the modulated and unmodulated parts with a chi-square fit after binning in energy and in 30-day time intervals (we fix the modulation phase to DAMA's best fit value of 152.5 days from January $1^{\text {st }}$ ). We use the acceptance shown in Fig. 20 of Ref. 62], parametrized as $C(E)=1-$ $\exp (-a E)$, with $E$ in keVee and $a=1.21$. As in [15, 17], in the figures we plot the unmodulated component of $\tilde{\eta}$ plus an unknown flat background $b_{0}$.

$C D M S-I I$. We use the germanium data (which we call CDMS-II-Ge) from the T1Z5 detector [10], which gives the most stringent limits at low WIMP masses. We compute the upper limit on $\tilde{\eta}^{0}$ using the maximum gap method [63] in the range $2 \mathrm{keV}-20 \mathrm{keV}$. We also include the CDMS-II 95\% upper bound of 0.045 events $/ \mathrm{kg} / \mathrm{day} / \mathrm{keV}$ on the rate modulation amplitude for a modulation phase equal to DAMA's in the energy range $5 \mathrm{keV}-11.9$ $\mathrm{keV}$ 11 and use Eq. (33) or Eq. (34) to find an upper limit on $\overline{v^{r} \tilde{\eta}_{\left[E_{1}^{\prime}, E_{2}^{\prime}\right]}^{1}}$ by imposing an upper limit on $\hat{R}_{\left[E_{1}^{\prime}, E_{2}^{\prime}\right]}^{1}$. In addition, we include the recent results from the silicon detector analysis in Ref. [5], which we denote as CDMS-II-Si. Since the energy resolution for silicon in CDMS-II has not been measured, we use the energy resolution for Ge in Eq. (1) of Ref. [64, $\sigma_{E_{\mathrm{R}}}(E)=\sqrt{0.293^{2}+0.056^{2} \mathrm{E} / \mathrm{keV}} \mathrm{keV}$. With three candidate events, we calculate the maximum gap upper limit by taking $\tilde{\eta}(v)$ as a downward step function as explained at the end of Sec. 2. Assuming the events are a DM signal, we bin the recoil spectrum in $2 \mathrm{keV}$ energy intervals, 7 to 9,9 to 11 and 11 to $13 \mathrm{keV}$, resulting in 1 event per bin. We use the Poisson central confidence interval of $(0.173,3.30)$ expected events for zero background at the $68 \%$ confidence level to draw error bars.

$X E N O N 100$. We use the last data release of Ref. [8], with total exposure of 224.6 days $\times 34 \mathrm{~kg}$. We derive the upper limits using the expressions described in Ref. 15]. We convert the energies of the two candidate events 

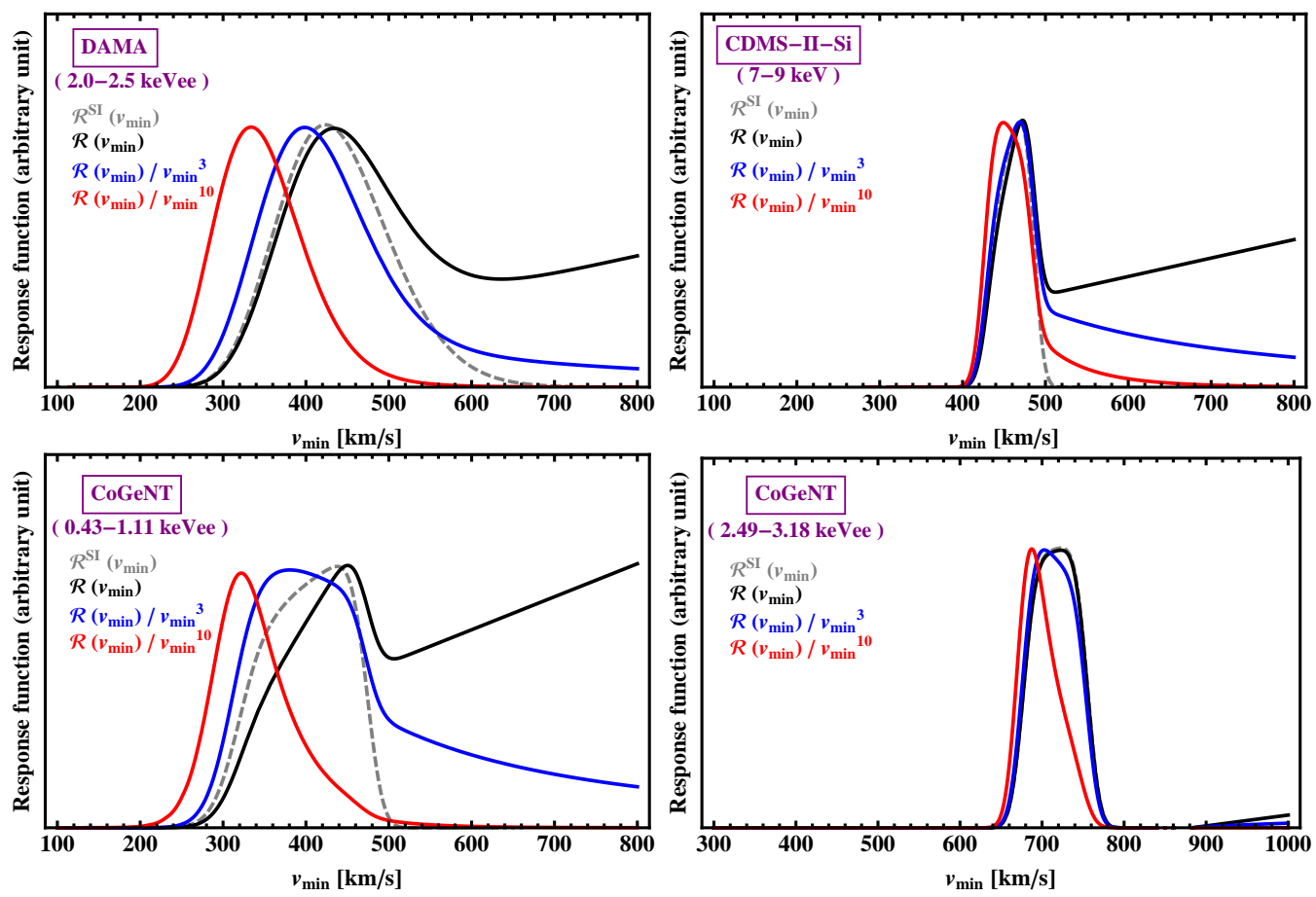

Figure 1: Response functions $v_{\min }^{-r} \mathcal{R}_{\left[E_{1}^{\prime}, E_{2}^{\prime}\right]}\left(v_{\min }\right)$ with arbitrary normalization for several detected energy intervals and detectors for SI interactions (gray dashed line) and for MDM with $m=9 \mathrm{GeV}$.

into $S 1$ values, and use the Poisson fluctuation formula Eq. (15) in [65] to compute the energy response function. We use the light efficiency function $\mathcal{L}_{\text {eff }}$ in Fig. 1 of [7] and the cut acceptances of Ref. [8]. We use the maximum gap method over the interval $3 \leqslant S 1 \leqslant 30$ photoelectrons.

$X E N O N 10$. We take the data from Ref. [6] and use only $S 2$ without $S 1 / S 2$ discrimination. The exposure is $1.2 \mathrm{~kg} \times 12.5$ days. We consider the 23 events within the $1.4 \mathrm{keV}-10 \mathrm{keV}$ acceptance box in the Phys. Rev. Lett. article (not the arXiv preprint, which had an $S 2$ window cut). We take a conservative acceptance of 0.94 . For the energy resolution, we convert the quoted energies into number of electrons $n_{e}=E Q_{y}(E)$, with $\mathcal{Q}_{y}(E)$ as in Eq. 1 of $[6]$ with $k=0.11$, and use the Poisson fluctuation formula in Eq. (15) of [65].

In Fig. 1 we illustrate the effect of various choices of $r$ on the response function $v_{\min }^{-r} \mathcal{R}_{\left[E_{1}^{\prime}, E_{2}^{\prime}\right]}\left(v_{\min }\right)$ for MDM for several energy bins and experiments: 


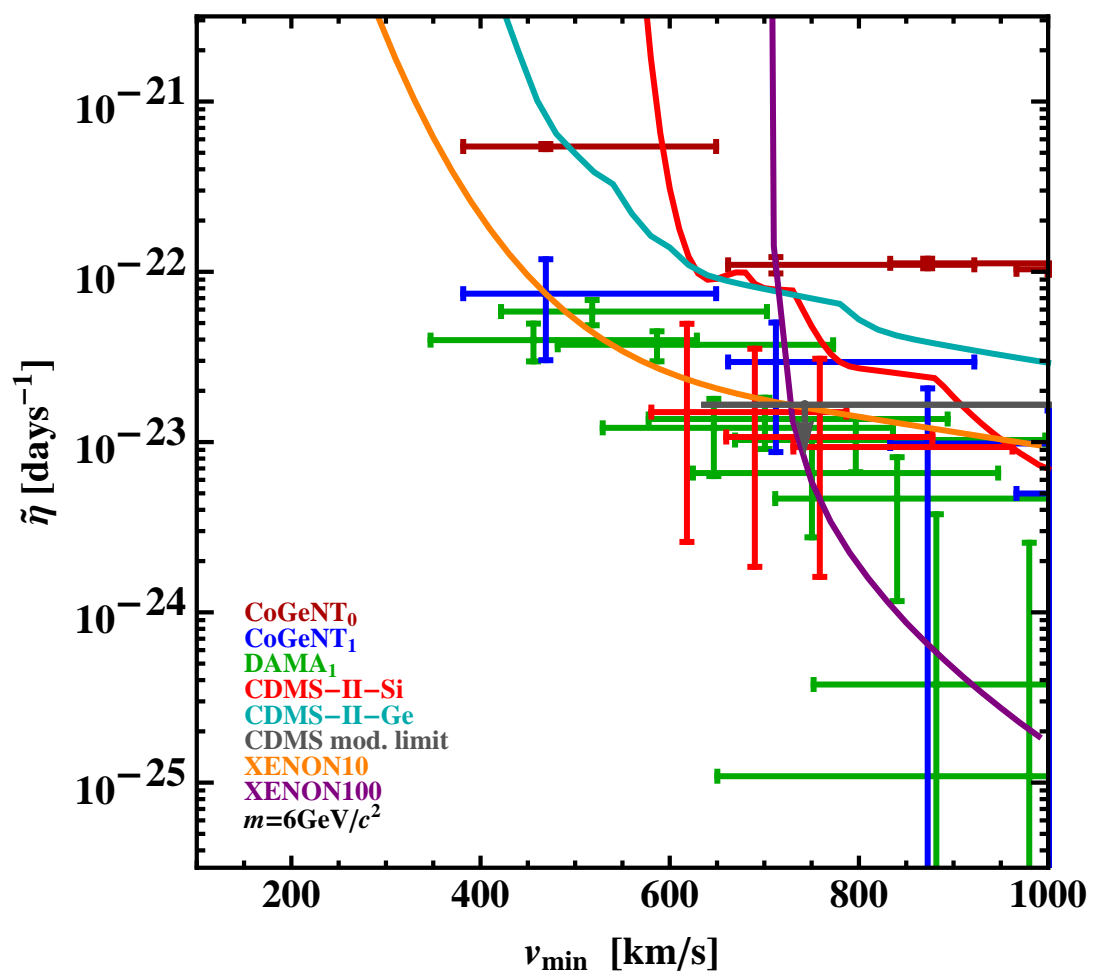

Figure 2: Measurements and bounds on $v_{\min }^{-10} \overline{v_{\min }^{10} \tilde{\eta}^{0}\left(v_{\min }\right)}$ and $v_{\min }^{-10} \overline{v_{\min }^{10} \tilde{\eta}^{1}\left(v_{\min }\right)}$ for a WIMP of mass $m=6 \mathrm{GeV}$ with magnetic dipole interactions (MDM). The vertical axis has the usual $\tilde{\eta}$ units of day ${ }^{-1}$.

the first energy bin of DAMA/LIBRA [1], 2 to $2.5 \mathrm{keVee}$, the 7 to $9 \mathrm{keV}$ CDMS-II used for the Si data [5 and the first, 0.43 to $1.11 \mathrm{keVee}$, and last, 2.49 to $3.18 \mathrm{keVee}$, of CoGeNT [2, 3. We also include $\mathcal{R}_{\left[E_{1}^{\prime}, E_{2}^{\prime}\right]}^{\mathrm{SI}}\left(v_{\min }\right)$ for the standard SI interaction (gray dashed line) for a comparison. The normalization of each curve is arbitrary. For $r=0$, the MDM response function is divergent and goes like $v$ at large velocities, given the $v^{2}$ behavior of $\left(v^{2} \mathrm{~d} \sigma_{T} / \mathrm{d} E_{\mathrm{R}}\right)$ (see discussion at the beginning of Sec. 44). The divergent behavior is much more pronounced in the low-energy bins. The choice $r=3$ is already enough to regularize the divergent behavior, but still yields too large $v_{\min }$ intervals. For growing values of $r$, the peak of the response function, mostly in the low energy bins, shifts towards low velocities, due to the $v^{-r}$ factor. This peak, when far from the $v_{\text {min }}$ interval where $\mathcal{R}_{\left[E_{1}^{\prime}, E_{2}^{\prime}\right]}(v)$ is nonnegligible, is unreliable as it is due to the low energy tail of the detector 


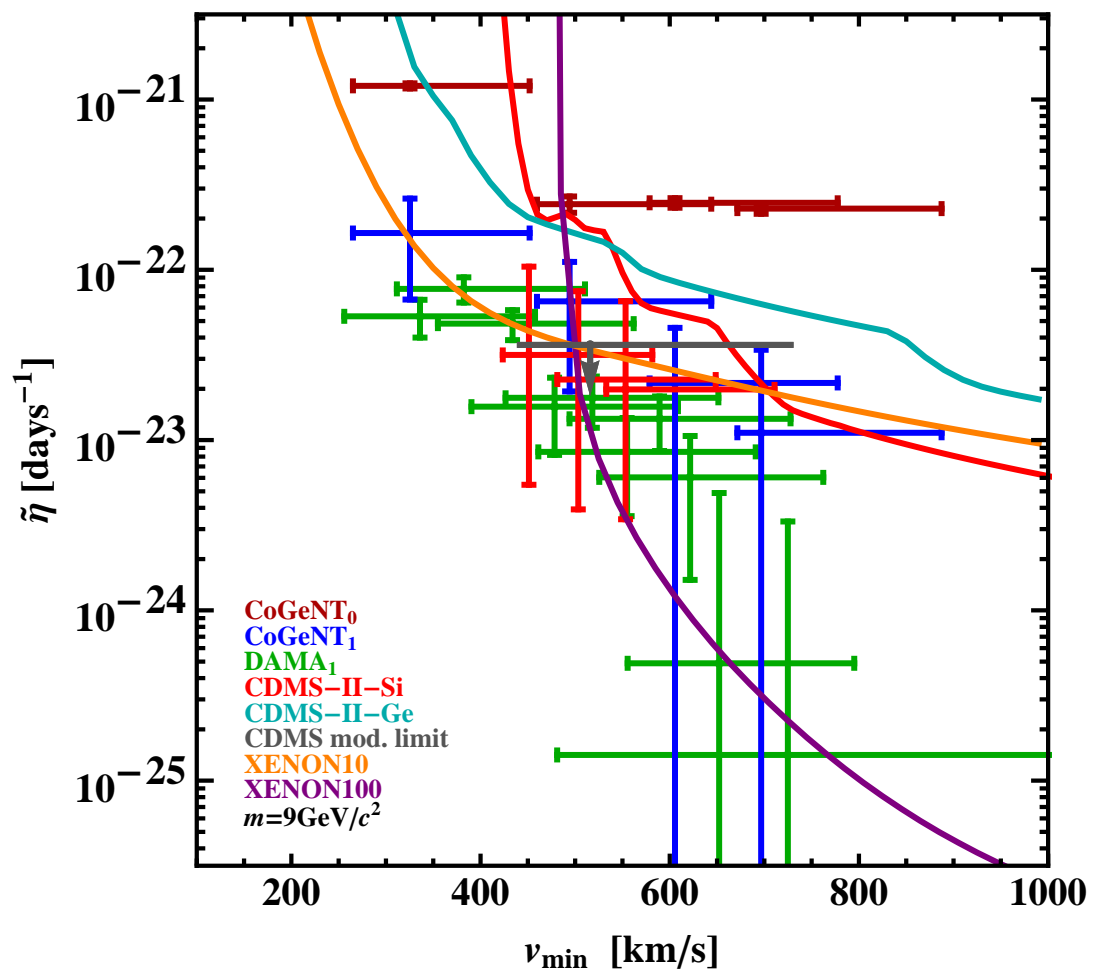

Figure 3: As Fig. 2 but for $m=9 \mathrm{GeV}$. All data points have moved to smaller $v_{\min }$ values as expected.

energy resolution function $G_{T}\left(E_{\mathrm{R}}, E^{\prime}\right)$, which determines the low velocity tail of $\mathcal{R}_{\left[E_{1}^{\prime}, E_{2}^{\prime}\right]}\left(v_{\min }\right)$ (see Eq. (20)) and is never well known. We found the optimum $r$ value by trial an error and for MDM we find that $r=10$ is an adequate choice (see Fig. 1) to get a localized response function in $v_{\min }$ space without relying on how the low energy tail of the energy resolution function is modeled. The choice of $r$ is dictated by the lowest energy bins, where the function $v^{-r}$ is largest. Higher energy bins are less sensitive to the choice of $r$.

Let us remark that this way of comparing data is not an inherent part to the halo independent method but only due to our choice of finding averages over measured energy bins to translate putative measurements of a DM signal. So far we have not found a better way of presenting the data, but more work is necessary to make progress in this respect.

Figs. 2, 3 and 4 show the measurements and bounds on $v_{\min }^{-10} \overline{v_{\min }^{10} \tilde{\eta}^{0}\left(v_{\text {min }}\right)}$ 


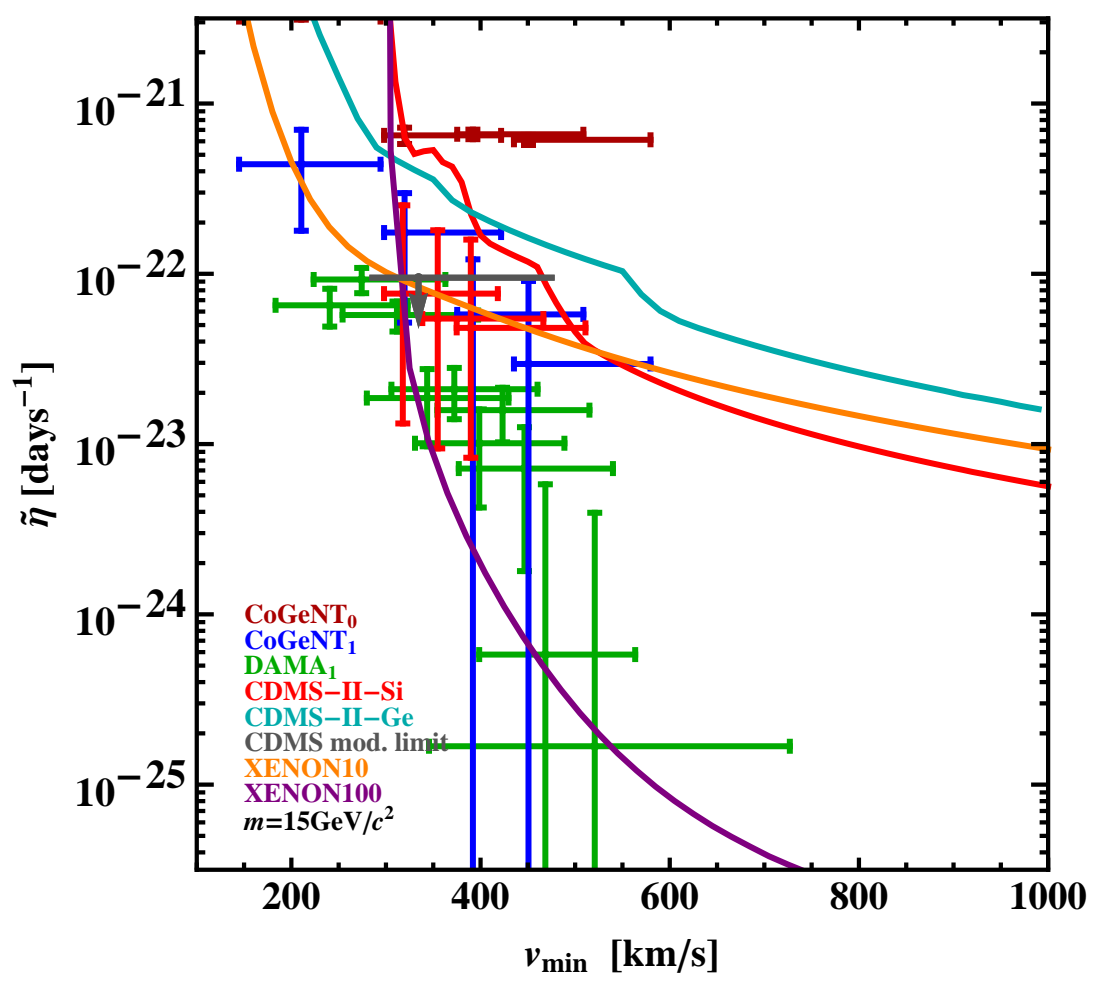

Figure 4: As Fig. 2 but for $m=15 \mathrm{GeV}$.

and $v_{\min }^{-10} \overline{v_{\min }^{10} \tilde{\eta}^{1}\left(v_{\min }\right)}$ for a WIMP with magnetic dipole interactions (MDM). To compute the position of the lines, no average is taken so that the bound corresponds to a limit on $\tilde{\eta}^{0}\left(v_{\min }\right)$.

In both figures we include the DAMA modulation signal (green crosses), CoGeNT modulated (blue crosses) and unmodulated signal (plus an unknown flat background, dark red horizontal lines), CDMS-II-Si unmodulated rate signal (red crosses and limit line), CDMS-II-Ge unmodulated rate limit (light blue line) and modulation bound (dark grey horizontal line), XENON100 225 days limit (purple line) and XENON10 S2 only limit (orange line).

Figs. 2, 3 and 4 differ for the value of the DM mass, respectively $m=6$ $\mathrm{GeV}, 9 \mathrm{GeV}$ and $15 \mathrm{GeV}$. We chose these masses motivated by previous studies on MDM as a potential explanation for the putative DM signal found by DAMA/LIBRA, CoGeNT and CRESST-II (see e.g. Ref. [48]). The measurements and limits for MDM move to larger $v_{\min }$ values as the WIMP mass increases, as expected due to the relation between $v_{\min }$ and the recoil 
energy. As shown in Fig. 2, for a WIMP of mass $m=6 \mathrm{GeV}$ the three CDMS-II-Si points are largely below the XENON10 and XENON100 upper limits, but they move progressively above them as $m$ increases to $9 \mathrm{GeV}$, see Fig. 3, and are almost entirely excluded by them for $m=15 \mathrm{GeV}$ in Fig. 4. The three CDMS-II-Si points overlap or are below the CoGeNT and DAMA/LIBRA measurements of the modulated part of $\tilde{\eta}$, except for the lowest energy CoGeNT and DAMA points. Thus, interpreted as a measurement of the unmodulated rate, the three CDMS-II-Si data points seem largely incompatible with the modulation of the signal observed by CoGeNT and DAMA for MDM. For all three WIMP masses shown in the figures, the DAMA and CoGeNT modulation measurements seem compatible with each other, but the upper limits on the unmodulated part of the rate imposed by XENON10 and XENON100 reject the DAMA/LIBRA and CoGeNT modulation signal, except for the lowest energy bins, for MDM.

\section{Conclusions}

We have presented a way to generalize to any DM-target nucleus interaction the halo-independent method to compare direct dark matter results from different experiments, initially proposed in Ref [13] and used already in several subsequent papers $[14-20]$. The method avoids the complications brought about by astrophysical uncertainties that affect the interaction rate.

The main idea of this method is that the interaction rate at one particular recoil energy $E_{\mathrm{R}}$ depends for any experiment on one and the same function $\eta\left(v_{\text {min }}\right)$ of the minimum speed $v_{\text {min }}$ required for the incoming DM particle to cause a nuclear recoil with energy $E_{\mathrm{R}}$. The function $\eta\left(v_{\min }\right)$ depends only on the local characteristics of the dark halo of our galaxy. Thus, all rate measurements and bounds can be translated into measurements and bounds on the unique function $\eta\left(v_{\text {min }}\right)$. Before the present work, this method was applied to the standard spin-independent (SI) WIMP-nucleus interaction only, although it could easily be applied to the standard spin-dependent (SD) interaction as well. For both SI and SD interactions, the differential scattering cross section has a $1 / v^{2}$ dependence on the speed $v$ of the DM particle. However, there are many other kinds of interactions with more general dependence on the DM particle velocity and on the nuclear recoil energy and for some of them the trivial extension of the SI method does not work. This is the case, for example when the cross section contains two 
different terms with different dependences on the DM particle speed $v$. Then, when these terms are integrated over the velocity distribution to find the rate, instead of a unique function $\eta\left(v_{\text {min }}\right)$, each term has its own function of $v_{\text {min }}$ multiplied by its own detector dependent coefficient. It is thus impossible to translate a rate measurement or bound into only one of the two $v_{\min }$ functions contributing to the rate.

In Eq. (26) we have presented a way to write the rate measured in a certain energy range $\left[E_{1}^{\prime}, E_{2}^{\prime}\right]$ (expressed in observed energy $E^{\prime}$, not in actual recoil energy $E_{R}$ ) for any kind of interaction in terms of a unique function of $v_{\text {min }}$, which we called $\tilde{\eta}$, that depends on the local characteristics of the dark halo of our galaxy only convolved with a detector and DM candidate dependent response function in $v_{\min }, \mathcal{R}_{\left[E_{1}^{\prime}, E_{2}^{\prime}\right]}$. This response function is defined in Eq. (27), as the derivative of what we call the "integrated response function" $\mathcal{H}_{\left[E_{1}^{\prime}, E_{2}^{\prime}\right]}$ defined in Eq. 20) or Eq. 21] in terms of the scattering cross section and detector characteristics (composition, energy resolution, efficiency cuts).

Since the function $\tilde{\eta}\left(v_{\text {min }}\right)$ must be common to all experiments, we can map all the rate measurements and bounds obtained with different experiments into the $\left(v_{\min }, \tilde{\eta}\right)$ plane, as in the case of SI interactions. We have then chosen a way to compare all data for magnetic dipole moment DM (MDM) by comparing weighted averages of the $\tilde{\eta}$ derived from experiments with a potential DM signal and upper bounds on $\tilde{\eta}$ derived from data which do not find a possible DM signal. The average is weighted by the response function $\mathcal{R}_{\left[E_{1}^{\prime}, E_{2}^{\prime}\right]}$ and corresponds to the $v_{\text {min }}$ interval in which this weight function is significantly different from zero. However, we found that for a differential cross section with a general dependence on the DM velocity the width of the response function $\mathcal{R}_{\left[E_{1}^{\prime}, E_{2}^{\prime}\right]}(v)$ in Eq. (27) at large $v_{\text {min }}$, which is dictated by the high speed behavior of the differential cross section, might even be infinite. For example, if $v^{2}\left(\mathrm{~d} \sigma_{T} / \mathrm{d} E_{\mathrm{R}}\right)$ goes as $v^{n}$, with $n$ a positive integer, for large $v$, then $\mathcal{H}_{\left[E_{1}^{\prime}, E_{2}^{\prime}\right]}(v)$ also goes as $v^{n}$ and $\mathcal{R}_{\left[E_{1}^{\prime}, E_{2}^{\prime}\right]}(v)$ goes as $v^{n-1}$ for large $v$. Thus, if $n \geqslant 1$, the response function $\mathcal{R}_{\left[E_{1}^{\prime}, E_{2}^{\prime}\right]}(v)$ does not vanish for large $v$. However, we can regularize the behavior of the response function at large $v$ by using for example the function $v^{r} \tilde{\eta}(v)$ with integer $r \geqslant n$, instead of just $\tilde{\eta}(v)$. Since this new function is common to all experiments, we can use it to compare the data in $v_{\text {min }}$ space. The power $r$ cannot be chosen arbitrarily large, because large values of $r$ give a large weight to the low velocity tail of the $\mathcal{R}_{\left[E_{1}^{\prime}, E_{2}^{\prime}\right]}(v)$ function, and this tail depends on the low energy tail of the experimental energy resolution function $G_{T}\left(E_{\mathrm{R}}, E^{\prime}\right)$ in Eq. (20), which is never well known. Therefore too large values of $r$ make the procedure 
very sensitive to the way in which the tails of the $G_{T}\left(E_{\mathrm{R}}, E^{\prime}\right)$ function are modeled. For the particular example of interaction we present in this paper, magnetic dipole DM or MDM, we found that an optimal choice is $r=10$. In the figures, rather than drawing the new averages $\overline{v^{r} \tilde{\eta}^{i}}$ and the limits $v^{r} \tilde{\eta}^{\lim }(v)$, we prefer to draw $v^{-r} \bar{v}^{r} \tilde{\eta}^{i}$ and $\tilde{\eta}^{\lim }(v)$, so that a comparison can be easily made with the previous literature on the SI halo-independent method.

Let us remark that this way of comparing data is not an inherent part to the halo independent method but only due to our choice of finding averages over measured energy bins to translate putative measurements of a DM signal. So far we have not found a better way of presenting the data, but more work is necessary to make progress in this respect.

\section{Acknowledgments}

P.G. was supported in part by NSF grant PHY-1068111. E.D.N., G.G. and J.-H.H. were supported in part by DOE grant DE-FG02-13ER42022. J.H.H. was also partially supported by Spanish Consolider-Ingenio MultiDark (CSD2009-00064).

\section{Appendix A - Inelastic scattering}

The DM particle may collide inelastically with the target nucleus [66], in which case the DM particle scatters to a different state with mass $m^{\prime}=$ $m+\delta$. Dark matter interacting inelastically via a magnetic dipole moment interaction [39,46] would require a modification of some of the equations presented above, in particular the definitions of $\mathcal{H}_{\left[E_{1}^{\prime}, E_{2}^{\prime}\right]}$. Here we present the relevant equations for the inelastic case.

In inelastic scattering, the minimum velocity the DM must have to impart a nuclear recoil energy $E_{\mathrm{R}}$ depends on the mass splitting $\delta$,

$$
v_{\min }=\frac{1}{\sqrt{2 m_{T} E_{\mathrm{R}}}}\left|\frac{m_{T} E_{\mathrm{R}}}{\mu_{T}}+\delta\right|,
$$

where $\delta$ can be either positive (endothermic scattering [66]) or negative (exothermic [67]) ( $\delta=0$ for elastic scattering). Inverting this equation implies the existence of both a maximum and a minimum recoil energy for a 
fixed DM velocity $v$ : $E_{\mathrm{R}}^{-}(v)<E_{\mathrm{R}}<E_{\mathrm{R}}^{+}(v)$, with

$$
E_{\mathrm{R}}^{ \pm}(v)=\frac{\mu_{T}^{2} v^{2}}{2 m_{T}}\left(1 \pm \sqrt{1-\frac{2 \delta}{\mu_{T} v^{2}}}\right)^{2} .
$$

The event rate in a detected energy interval $\left[E_{1}^{\prime}, E_{2}^{\prime}\right]$ is (as in Eq. (40)

$$
\begin{array}{r}
R_{\left[E_{1}^{\prime}, E_{2}^{\prime}\right]}(t)=\frac{\rho}{m} \sum_{T} \frac{C_{T}}{m_{T}} \int_{0}^{\infty} \mathrm{d} E_{\mathrm{R}} \int_{v \geqslant v_{\min }\left(E_{\mathrm{R}}\right)} \mathrm{d}^{3} v f(\mathbf{v}, t) v \frac{\mathrm{d} \sigma_{T}}{\mathrm{~d} E_{\mathrm{R}}}\left(E_{\mathrm{R}}, \mathbf{v}\right) \\
\times \int_{E_{1}^{\prime}}^{E_{2}^{\prime}} \mathrm{d} E^{\prime} \epsilon\left(E^{\prime}\right) G_{T}\left(E_{\mathrm{R}}, E^{\prime}\right) .
\end{array}
$$

Changing the order of the integrations in $\mathbf{v}$ and $E_{\mathrm{R}}$ in Eq. (44), we have

$$
\begin{aligned}
R_{\left[E_{1}^{\prime}, E_{2}^{\prime}\right]}(t)=\frac{\rho \sigma_{\mathrm{ref}}}{m} \int_{v \geqslant \hat{v}_{\delta}} \mathrm{d}^{3} v \frac{f(\mathbf{v}, t)}{v} \sum_{T} \frac{C_{T}}{m_{T}} \int_{E_{\mathrm{R}}^{-}(v)}^{E_{\mathrm{R}}^{+}(v)} \mathrm{d} E_{\mathrm{R}} \frac{v^{2}}{\sigma_{\mathrm{ref}}} \frac{\mathrm{d} \sigma_{T}}{\mathrm{~d} E_{\mathrm{R}}}\left(E_{\mathrm{R}}, \mathbf{v}\right) \\
\times \int_{E_{1}^{\prime}}^{E_{2}^{\prime}} \mathrm{d} E^{\prime} \epsilon\left(E^{\prime}\right) G_{T}\left(E_{\mathrm{R}}, E^{\prime}\right),
\end{aligned}
$$

where $\hat{v}_{\delta}$ is the minimum value $v_{\min }$ can take, $\hat{v}_{\delta}=\sqrt{2 \delta / \mu_{T}}$ for $\delta>0$ and $\hat{v}_{\delta}=0$ for $\delta \leqslant 0$. In compact form, Eq. 45 reads

$$
R_{\left[E_{1}^{\prime}, E_{2}^{\prime}\right]}(t)=\int_{v \geqslant \hat{v}_{\delta}} \mathrm{d}^{3} v \frac{\tilde{f}(\mathbf{v}, t)}{v} \mathcal{H}_{\left[E_{1}^{\prime}, E_{2}^{\prime}\right]}(\mathbf{v}),
$$

where as in Eq. (19)

$$
\tilde{f}(\mathbf{v}, t) \equiv \frac{\rho \sigma_{\text {ref }}}{m} f(\mathbf{v}, t)
$$

and

$$
\begin{aligned}
\mathcal{H}_{\left[E_{1}^{\prime}, E_{2}^{\prime}\right]}(\mathbf{v}) \equiv \sum_{T} \frac{C_{T}}{m_{T}} \int_{E_{\mathrm{R}}^{-}(v)}^{E_{\mathrm{R}}^{+}(v)} \mathrm{d} E_{\mathrm{R}} \frac{v^{2}}{\sigma_{\mathrm{ref}}} & \frac{\mathrm{d} \sigma_{T}}{\mathrm{~d} E_{\mathrm{R}}}\left(E_{\mathrm{R}}, \mathbf{v}\right) \\
& \times \int_{E_{1}^{\prime}}^{E_{2}^{\prime}} \mathrm{d} E^{\prime} \epsilon\left(E^{\prime}\right) G_{T}\left(E_{\mathrm{R}}, E^{\prime}\right) .
\end{aligned}
$$

We will deal in detail with the halo independent comparison of direct detection data for dark matter with magnetic dipole interactions (MDM) scattering inelastically $[39,46]$ elsewhere. 


\section{Appendix B - Rate in terms of partial moments}

In this appendix we derive Eqs. (29) and (31). Define, as in Eq. (28),

$$
\begin{aligned}
& \tilde{\eta}_{(0)}(v) \equiv \tilde{\eta}(v)=\int_{v_{\min }}^{\infty} \mathrm{d} v \frac{\tilde{F}(v)}{v}, \\
& \tilde{\eta}_{(k)}(v) \equiv k \int_{v}^{\infty} \mathrm{d} v^{\prime} \tilde{\eta}_{(k-1)}\left(v^{\prime}\right), \quad \text { for integer } k>0 .
\end{aligned}
$$

Then

$$
\tilde{\eta}_{(k-1)}(v)=-\frac{1}{k} \frac{\partial \tilde{\eta}_{(k)}(v)}{\partial v}
$$

Repeatedly integrating by parts Eq. 26) gives

$$
\begin{aligned}
R_{\left[E_{1}^{\prime}, E_{2}^{\prime}\right]} & =\int_{0}^{\infty} \mathrm{d} v \tilde{\eta}(v) \mathcal{R}_{\left[E_{1}^{\prime}, E_{2}^{\prime}\right]}(v)=\int_{0}^{\infty} \mathrm{d} v \tilde{\eta}_{(0)}(v) \mathcal{R}_{\left[E_{1}^{\prime}, E_{2}^{\prime}\right]}(v) \\
& =-\int_{0}^{\infty} \mathrm{d} v \frac{\partial \tilde{\eta}_{(1)}(v)}{\partial v} \mathcal{R}_{\left[E_{1}^{\prime}, E_{2}^{\prime}\right]}(v)=\int_{0}^{\infty} \mathrm{d} v \tilde{\eta}_{(1)}(v) \frac{\partial \mathcal{R}_{\left[E_{1}^{\prime}, E_{2}^{\prime}\right]}(v)}{\partial v} \\
& =-\frac{1}{2} \int_{0}^{\infty} \mathrm{d} v \frac{\partial \tilde{\eta}_{(2)}(v)}{\partial v} \frac{\partial \mathcal{R}_{\left[E_{1}^{\prime}, E_{2}^{\prime}\right]}(v)}{\partial v}=\frac{1}{2} \int_{0}^{\infty} \mathrm{d} v \tilde{\eta}_{(2)}(v) \frac{\partial^{2} \mathcal{R}_{\left[E_{1}^{\prime}, E_{2}^{\prime}\right]}(v)}{\partial v^{2}} \\
& =\cdots \\
& =\frac{1}{k !} \int_{0}^{\infty} \mathrm{d} v \tilde{\eta}_{(k)}(v) \frac{\partial^{k} \mathcal{R}_{\left[E_{1}^{\prime}, E_{2}^{\prime}\right]}(v)}{\partial v^{k}} .
\end{aligned}
$$

In deriving this result, all boundary terms vanish because we have assumed that the response function and all of its derivatives vanish at $v=0$, since $v=0$ is below the threshold of any experiment.

Notice that we can write each $\tilde{\eta}_{(k)}\left(v_{\min }\right)$ in terms of a single integral of $\widetilde{F}(v) / v$ as

$$
\tilde{\eta}_{(k)}\left(v_{\min }\right)=\int_{v_{\min }}^{\infty} \mathrm{d} v\left(v-v_{\min }\right)^{k} \frac{\widetilde{F}(v)}{v} .
$$


This follows by induction from Eq. (49) and

$$
\begin{aligned}
\tilde{\eta}_{(k)}(v) & =k \int_{v}^{\infty} \mathrm{d} v_{2} \tilde{\eta}_{(k-1)}\left(v_{2}\right)=k \int_{v}^{\infty} \mathrm{d} v_{2} \int_{v_{2}}^{\infty} \mathrm{d} v_{1}\left(v_{1}-v_{2}\right)^{k-1} \frac{\tilde{F}\left(v_{1}\right)}{v_{1}} \\
& =k \int_{v}^{\infty} \mathrm{d} v_{1} \int_{v}^{v_{1}} \mathrm{~d} v_{2}\left(v_{1}-v_{2}\right)^{k-1} \frac{\tilde{F}\left(v_{1}\right)}{v_{1}}=k \int_{v}^{\infty} \mathrm{d} v_{1} \int_{0}^{v_{1}-v} \mathrm{~d} v_{3} v_{3}^{k-1} \frac{\tilde{F}\left(v_{1}\right)}{v_{1}} \\
& =\int_{v}^{\infty} \mathrm{d} v_{1}\left[v_{3}^{k}\right]_{0}^{v_{1}-v} \frac{\tilde{F}\left(v_{1}\right)}{v_{1}}=\int_{v}^{\infty} \mathrm{d} v_{1}\left(v_{1}-v\right)^{k} \frac{\tilde{F}\left(v_{1}\right)}{v_{1}}
\end{aligned}
$$

In terms of the velocity distribution $\tilde{f}(\mathbf{v})$, we see now that $\tilde{\eta}_{(k)}\left(v_{\min }\right)$ is the so-called $k$-th partial moment of the function $\tilde{f}(\mathbf{v}) / v$, as in Eq. (31) above,

$$
\tilde{\eta}_{(k)}\left(v_{\text {min }}\right)=\int_{v \geqslant v_{\min }}\left(v-v_{\min }\right)^{k} \frac{\tilde{f}(\mathbf{v})}{v} \mathrm{~d}^{3} v .
$$

\section{References}

[1] R. Bernabei et al. [DAMA and LIBRA Collaborations], "New results from DAMA/LIBRA," Eur. Phys. J. C 67 (2010) 39 arXiv:1002.1028 [astro-ph.GA]].

[2] C. E. Aalseth et al. [CoGeNT Collaboration], "Results from a Search for Light-Mass Dark Matter with a P-type Point Contact Germanium Detector," Phys. Rev. Lett. 106 (2011) 131301 arXiv:1002.4703 [astroph.CO]].

[3] C. E. Aalseth, P. S. Barbeau, J. Colaresi, J. I. Collar, J. Diaz Leon, J. E. Fast, N. Fields and T. W. Hossbach et al., "Search for an Annual Modulation in a P-type Point Contact Germanium Dark Matter Detector," Phys. Rev. Lett. 107 (2011) 141301 arXiv:1106.0650 [astroph.CO]].

[4] G. Angloher, M. Bauer, I. Bavykina, A. Bento, C. Bucci, C. Ciemniak, G. Deuter and F. von Feilitzsch et al., "Results from $730 \mathrm{~kg}$ days of the CRESST-II Dark Matter Search," Eur. Phys. J. C 72 (2012) 1971 arXiv:1109.0702 [astro-ph.CO]].

[5] R. Agnese et al. [CDMS Collaboration], "Dark Matter Search Results Using the Silicon Detectors of CDMS II," arXiv:1304.4279 [hep-ex]]. 
[6] J. Angle et al. [XENON10 Collaboration], "A search for light dark matter in XENON10 data," Phys. Rev. Lett. 107 (2011) 051301 arXiv:1104.3088 [astro-ph.CO]].

[7] E. Aprile et al. [XENON100 Collaboration], "Dark Matter Results from 100 Live Days of XENON100 Data," Phys. Rev. Lett. 107 (2011) 131302 arXiv:1104.2549 [astro-ph.CO]].

[8] E. Aprile et al. [XENON100 Collaboration], "Dark Matter Results from 225 Live Days of XENON100 Data," Phys. Rev. Lett. 109 (2012) 181301 arXiv:1207.5988 [astro-ph.CO]].

[9] M. Felizardo, T. A. Girard, T. Morlat, A. C. Fernandes, A. R. Ramos, J. G. Marques, A. Kling and J. Puibasset et al., "Final Analysis and Results of the Phase II SIMPLE Dark Matter Search," Phys. Rev. Lett. 108 (2012) 201302 arXiv:1106.3014 [astro-ph.CO]].

[10] Z. Ahmed et al. [CDMS-II Collaboration], "Results from a Low-Energy Analysis of the CDMS II Germanium Data," Phys. Rev. Lett. 106 (2011) 131302 arXiv:1011.2482 [astro-ph.CO]].

[11] Z. Ahmed et al. [CDMS Collaboration], "Search for annual modulation in low-energy CDMS-II data," arXiv:1203.1309 [astro-ph.CO].

[12] R. Agnese et al. [CDMS Collaboration], "Silicon Detector Results from the First Five-Tower Run of CDMS II," arXiv:1304.3706 [astroph.CO]].

[13] P. J. Fox, J. Liu and N. Weiner, "Integrating Out Astrophysical Uncertainties," Phys. Rev. D 83 (2011) 103514 arXiv:1011.1915 [hep-ph]].

[14] M. T. Frandsen, F. Kahlhoefer, C. McCabe, S. Sarkar and K. SchmidtHoberg, "Resolving astrophysical uncertainties in dark matter direct detection," JCAP 1201 (2012) 024 arXiv:1111.0292 [hep-ph]].

[15] P. Gondolo and G. B. Gelmini, "Halo independent comparison of direct dark matter detection data," JCAP 1212 (2012) 015 arXiv:1202.6359 [hep-ph]].

[16] M. T. Frandsen, F. Kahlhoefer, C. McCabe, S. Sarkar and K. SchmidtHoberg, "The unbearable lightness of being: CDMS versus XENON," arXiv:1304.6066 [hep-ph]. 
[17] E. Del Nobile, G. B. Gelmini, P. Gondolo and J. -H. Huh, "Haloindependent analysis of direct detection data for light WIMPs," arXiv:1304.6183 [hep-ph].

[18] J. Herrero-Garcia, T. Schwetz and J. Zupan, "On the annual modulation signal in dark matter direct detection," JCAP 1203 (2012) 005 arXiv:1112.1627 [hep-ph]].

[19] J. Herrero-Garcia, T. Schwetz and J. Zupan, "Astrophysics independent bounds on the annual modulation of dark matter signals," Phys. Rev. Lett. 109 (2012) 141301 arXiv:1205.0134 [hep-ph]].

[20] N. Bozorgnia, J. Herrero-Garcia, T. Schwetz and J. Zupan, "Halo-independent methods for inelastic dark matter scattering," arXiv:1305.3575 [hep-ph].

[21] J. Bagnasco, M. Dine and S. D. Thomas, "Detecting technibaryon dark matter," Phys. Lett. B 320 (1994) 99 hep-ph/9310290.

[22] A. Kurylov and M. Kamionkowski, "Generalized analysis of weakly interacting massive particle searches," Phys. Rev. D 69 (2004) 063503 hep-ph/0307185.

[23] B. A. Dobrescu and I. Mocioiu, "Spin-dependent macroscopic forces from new particle exchange," JHEP 0611 (2006) 005 hep-ph/0605342.

[24] J. Fan, M. Reece and L. -T. Wang, "Non-relativistic effective theory of dark matter direct detection," JCAP 1011 (2010) 042 arXiv:1008.1591 [hep-ph]].

[25] A. L. Fitzpatrick, W. Haxton, E. Katz, N. Lubbers and Y. Xu, "The Effective Field Theory of Dark Matter Direct Detection," JCAP 1302 (2013) 004 [arXiv:1203.3542 [hep-ph]].

[26] M. Beltran, D. Hooper, E. W. Kolb and Z. C. Krusberg, "Deducing the nature of dark matter from direct and indirect detection experiments in the absence of collider signatures of new physics," Phys. Rev. D 80 (2009) 043509 arXiv:0808.3384 [hep-ph]].

[27] S. Chang, A. Pierce and N. Weiner, "Momentum Dependent Dark Matter Scattering," JCAP 1001 (2010) 006 arXiv:0908.3192 [hep-ph]]. 
[28] J. -M. Zheng, Z. -H. Yu, J. -W. Shao, X. -J. Bi, Z. Li and H. -H. Zhang, "Constraining the interaction strength between dark matter and visible matter: I. fermionic dark matter," Nucl. Phys. B 854 (2012) 350 arXiv:1012.2022 [hep-ph]].

[29] Z. -H. Yu, J. -M. Zheng, X. -J. Bi, Z. Li, D. -X. Yao and H. -H. Zhang, "Constraining the interaction strength between dark matter and visible matter: II. scalar, vector and spin-3/2 dark matter," Nucl. Phys. B 860 (2012) 115 [arXiv:1112.6052 [hep-ph]].

[30] K. Cheung, P. -Y. Tseng, Y. -L. S. Tsai and T. -C. Yuan, "Global Constraints on Effective Dark Matter Interactions: Relic Density, Direct Detection, Indirect Detection, and Collider," JCAP 1205 (2012) 001 arXiv:1201.3402 [hep-ph]].

[31] J. March-Russell, J. Unwin and S. M. West, "Closing in on Asymmetric Dark Matter I: Model independent limits for interactions with quarks," JHEP 1208 (2012) 029 [arXiv:1203.4854 [hep-ph]].

[32] R. Ding and Y. Liao, "Spin 3/2 Particle as a Dark Matter Candidate: an Effective Field Theory Approach," JHEP 1204 (2012) 054 arXiv:1201.0506 [hep-ph]].

[33] M. Pospelov and T. ter Veldhuis, "Direct and indirect limits on the electromagnetic form-factors of WIMPs," Phys. Lett. B 480 (2000) 181 hep-ph/0003010.

[34] A. L. Fitzpatrick and K. M. Zurek, "Dark Moments and the DAMACoGeNT Puzzle," Phys. Rev. D 82 (2010) 075004 arXiv:1007.5325 [hep-ph]].

[35] H. An, S. -L. Chen, R. N. Mohapatra, S. Nussinov and Y. Zhang, "Energy Dependence of Direct Detection Cross Section for Asymmetric Mirror Dark Matter," Phys. Rev. D 82 (2010) 023533 arXiv:1004.3296 [hep-ph]].

[36] S. D. McDermott, H. -B. Yu and K. M. Zurek, "Turning off the Lights: How Dark is Dark Matter?," Phys. Rev. D 83 (2011) 063509 arXiv:1011.2907 [hep-ph]]. 
[37] K. Sigurdson, M. Doran, A. Kurylov, R. R. Caldwell and M. Kamionkowski, "Dark-matter electric and magnetic dipole moments," Phys. Rev. D 70 (2004) 083501 [Erratum-ibid. D 73 (2006) 089903] astro-ph/0406355].

[38] V. Barger, W. -Y. Keung and D. Marfatia, "Electromagnetic properties of dark matter: Dipole moments and charge form factor," Phys. Lett. B 696 (2011) 74 arXiv:1007.4345 [hep-ph]].

[39] S. Chang, N. Weiner and I. Yavin, "Magnetic Inelastic Dark Matter," Phys. Rev. D 82 (2010) 125011 arXiv:1007.4200 [hep-ph]].

[40] W. S. Cho, J. -H. Huh, I. -W. Kim, J. E. Kim and B. Kyae, "Constraining WIMP magnetic moment from CDMS II experiment," Phys. Lett. B 687 (2010) 6 [Erratum-ibid. B 694 (2011) 496] |arXiv:1001.0579 [hep-ph]].

[41] J. H. Heo, "Minimal Dirac Fermionic Dark Matter with Nonzero Magnetic Dipole Moment," Phys. Lett. B 693 (2010) 255 arXiv:0901.3815 [hep-ph]].

[42] S. Gardner, "Shedding Light on Dark Matter: A Faraday Rotation Experiment to Limit a Dark Magnetic Moment," Phys. Rev. D 79 (2009) 055007 arXiv:0811.0967 [hep-ph]].

[43] E. Masso, S. Mohanty and S. Rao, "Dipolar Dark Matter," Phys. Rev. D 80 (2009) 036009 arXiv:0906.1979 [hep-ph]].

[44] T. Banks, J. -F. Fortin and S. Thomas, "Direct Detection of Dark Matter Electromagnetic Dipole Moments," arXiv:1007.5515 [hep-ph].

[45] J. -F. Fortin and T. M. P. Tait, "Collider Constraints on Dipole-Interacting Dark Matter," Phys. Rev. D 85 (2012) 063506 arXiv:1103.3289 [hep-ph]].

[46] K. Kumar, A. Menon and T. M. P. Tait, "Magnetic Fluffy Dark Matter," JHEP 1202 (2012) 131 arXiv:1111.2336 [hep-ph]].

[47] V. Barger, W. -Y. Keung, D. Marfatia and P. -Y. Tseng, "Dipole Moment Dark Matter at the LHC," Phys. Lett. B 717 (2012) 219 arXiv:1206.0640 [hep-ph]]. 
[48] E. Del Nobile, C. Kouvaris, P. Panci, F. Sannino and J. Virkajarvi, "Light Magnetic Dark Matter in Direct Detection Searches," JCAP 1208 (2012) 010 arXiv:1203.6652 [hep-ph]].

[49] J. M. Cline, Z. Liu and W. Xue, "Millicharged Atomic Dark Matter," Phys. Rev. D 85 (2012) 101302 arXiv:1201.4858 [hep-ph]].

[50] N. Weiner and I. Yavin, "How Dark Are Majorana WIMPs? Signals from MiDM and Rayleigh Dark Matter," Phys. Rev. D 86 (2012) 075021 arXiv:1206.2910 [hep-ph]].

[51] S. Tulin, H. -B. Yu and K. M. Zurek, "Three Exceptions for Thermal Dark Matter with Enhanced Annihilation to $\gamma \gamma$," Phys. Rev. D 87 (2013) 036011 arXiv:1208.0009 [hep-ph]].

[52] J. M. Cline, A. R. Frey and G. D. Moore, "Composite magnetic dark matter and the $130 \mathrm{GeV}$ line," Phys. Rev. D 86 (2012) 115013 arXiv:1208.2685 [hep-ph]].

[53] M. Pospelov and A. Ritz, "Resonant scattering and recombination of pseudo-degenerate WIMPs," Phys. Rev. D 78 (2008) 055003 arXiv:0803.2251 [hep-ph]].

[54] Y. Bai and P. J. Fox, "Resonant Dark Matter," JHEP 0911 (2009) 052 arXiv:0909.2900 [hep-ph]].

[55] B. Feldstein, A. L. Fitzpatrick and E. Katz, "Form Factor Dark Matter," JCAP 1001 (2010) 020 [arXiv:0908.2991 [hep-ph]].

[56] C. M. Ho and R. J. Scherrer, "Anapole Dark Matter," Phys. Lett. B 722 (2013) 341 arXiv:1211.0503 [hep-ph]].

[57] R. H. Helm, "Inelastic and Elastic Scattering of 187-Mev Electrons from Selected Even-Even Nuclei," Phys. Rev. 104 (1956) 1466.

[58] T. W. Donnelly and I. Sick, "Elastic Magnetic Electron Scattering From Nuclei," Rev. Mod. Phys. 56 (1984) 461.

[59] N. Bozorgnia, G. B. Gelmini and P. Gondolo, "Channeling in direct dark matter detection I: channeling fraction in $\mathrm{NaI}(\mathrm{Tl})$ crystals," JCAP 1011 (2010) 019 arXiv:1006.3110 [astro-ph.CO]]. 
[60] J. I. Collar, "Quenching and Channeling of Nuclear Recoils in NaI[Tl]: Implications for Dark Matter Searches," arXiv:1302.0796 [physics.insdet].

[61] J. Collar, private communication.

[62] C. E. Aalseth et al. [CoGeNT Collaboration], "CoGeNT: A Search for Low-Mass Dark Matter using p-type Point Contact Germanium Detectors," Physical Review D 88, 012002 (2013) arXiv:1208.5737 [astro-ph.CO]].

[63] S. Yellin, "Finding an upper limit in the presence of unknown background," Phys. Rev. D 66 (2002) 032005 physics/0203002.

[64] Z. Ahmed et al. [CDMS Collaboration], "Analysis of the low-energy electron-recoil spectrum of the CDMS experiment," Phys. Rev. D 81 (2010) 042002 arXiv:0907.1438 [astro-ph.GA]].

[65] E. Aprile et al. [XENON100 Collaboration], "Likelihood Approach to the First Dark Matter Results from XENON100," Phys. Rev. D 84 (2011) 052003 arXiv:1103.0303 [hep-ex]].

[66] D. Tucker-Smith and N. Weiner, "Inelastic dark matter," Phys. Rev. D 64 (2001) 043502 hep-ph/0101138.

[67] P. W. Graham, R. Harnik, S. Rajendran and P. Saraswat, "Exothermic Dark Matter," Phys. Rev. D 82 (2010) 063512 [arXiv:1004.0937 [hep$\mathrm{ph}]$. 